\title{
A WEAK LENSING STUDY OF X-RAY GROUPS IN THE COSMOS SURVEY: FORM AND EVOLUTION OF THE MASS-LUMINOSITY RELATION*
}

\author{
Alexie Leauthaud $^{1,2}$, Alexis Finoguenov ${ }^{3,4}$, Jean-Paul KneiB ${ }^{5}$, James E. Taylor ${ }^{6}$, Richard Massey ${ }^{7}$, Jason Rhodes ${ }^{8,9}$, \\ Olivier Ilbert $^{5}$, Kevin Bundy $^{10,23}$, Jeremy Tinker ${ }^{2}$, Matthew R. GeOrge $^{10}$, Peter CaPaK $^{11}$, Anton M. Koekemoer $^{12}$, \\ David E. Johnston ${ }^{13}$, Yu-Ying Zhang ${ }^{14}$, Nico Cappelluti ${ }^{3}$, Richard S. Ellis ${ }^{9}$, Martin Elvis ${ }^{15}$, Stefania Giodini ${ }^{3}$, \\ Catherine Heymans ${ }^{7}$, Oliver Le Fèvre ${ }^{5}$, Simon Lilly ${ }^{16}$, Henry J. McCracken ${ }^{17}$, Yannick Mellier ${ }^{17}$, \\ Alexandre Réfrégier $^{18}$, Mara Salvato ${ }^{9,19}$, Nick Scoville ${ }^{9}$, George Smoot $^{1,2}$, Masayuki Tanaka ${ }^{20}$, \\ Ludovic VAN WAERBEKe ${ }^{21}$, AND MELODY WOLK 22 \\ ${ }^{1}$ Lawrence Berkeley National Laboratory, 1 Cyclotron Road, Berkeley CA 94720, USA; asleauthaud@1bl.gov \\ ${ }^{2}$ Berkeley Center for Cosmological Physics, University of California, Berkeley, CA 94720, USA \\ ${ }^{3}$ Max Planck Institut für extraterrestrische Physik, Giessenbachstrasse, D-85748 Garchingbei München, Germany \\ ${ }^{4}$ University of Maryland Baltimore County, 1000 Hilltop circle, Baltimore, MD 21250, USA \\ ${ }^{5}$ LAM, CNRS-UNiv Aix-Marseille, 38 rue F. Joliot-Curis, 13013 Marseille, France \\ ${ }^{6}$ Department of Physics and Astronomy, University of Waterloo, 200 University Avenue West, Waterloo, ON N2L 3G1, Canada \\ ${ }^{7}$ Institute for Astronomy, Blackford Hill, Edinburgh EH9 3HJ, UK \\ ${ }^{8}$ Jet Propulsion Laboratory, California Institute of Technology, Pasadena, CA 91109, USA \\ ${ }^{9}$ California Institute of Technology, MC 105-24, 1200 East California Boulevard, Pasadena, CA 91125, USA \\ ${ }^{10}$ Department of Astronomy, University of California, Berkeley, CA 94720, USA \\ ${ }^{11}$ Spitzer Science Center, 314-6 Caltech, 1201 E. California Blvd. Pasadena, CA 91125, USA \\ ${ }^{12}$ Space Telescope Science Institute, 3700 San Martin Drive, Baltimore, MD 21218, USA \\ ${ }^{13}$ Department of Physics \& Astronomy, Northwestern University, Evanston, IL 60208-2900, USA \\ ${ }^{14}$ Argelander Institut für Astronomie, Universität Bonn, Auf dem Hügel 71, 53121 Bonn, Germany \\ ${ }^{15}$ Harvard-Smithsonian Center for Astrophysics 60 Garden St., Cambridge, MA 02138, USA \\ ${ }^{16}$ Institute of Astronomy, Department of Physics, ETH Zurich, CH-8093, Switzerland \\ ${ }^{17}$ Institut d'Astrophysique de Paris, UMR 7095, 98 bis Boulevard Arago, 75014 Paris, France \\ ${ }^{18}$ Service d'Astrophysique, CEA/Saclay, 91191 Gif-sur-Yvette, France \\ ${ }^{19}$ IPP-Max Planck Institute for Plasma Physics, Boltzmann Strasse 2 Garching 85748, Germany \\ ${ }^{20}$ European Southern Observatory, Karl-Schwarzschild-Str. 2, 85748 Garching bei Munchen, Germany \\ ${ }^{21}$ Department of Physics \& Astronomy, University of British Columbia, 6224 Agricultural Road, Vancouver, BC V6T 1Z1, Canada \\ ${ }^{22}$ Ecole normale supèrieure de Cachan, 61 avenue du Président Wilson, 94235, Cachan cedex, France \\ Received 2009 May 9; accepted 2009 November 12; published 2009 December 29
}

\begin{abstract}
Measurements of X-ray scaling laws are critical for improving cosmological constraints derived with the halo mass function and for understanding the physical processes that govern the heating and cooling of the intracluster medium. In this paper, we use a sample of $206 \mathrm{X}$-ray-selected galaxy groups to investigate the scaling relation between X-ray luminosity $\left(L_{X}\right)$ and halo mass $\left(M_{200}\right)$ where $M_{200}$ is derived via stacked weak gravitational lensing. This work draws upon a broad array of multi-wavelength COSMOS observations including 1.64 degrees $^{2}$ of contiguous imaging with the Advanced Camera for Surveys to a limiting magnitude of $I_{\mathrm{F} 814 \mathrm{~W}}=26.5$ and deep XMM-Newton/Chandra imaging to a limiting flux of $1.0 \times 10^{-15} \mathrm{erg} \mathrm{cm}^{-2} \mathrm{~s}^{-1}$ in the $0.5-2 \mathrm{keV}$ band. The combined depth of these two data sets allows us to probe the lensing signals of X-ray-detected structures at both higher redshifts and lower masses than previously explored. Weak lensing profiles and halo masses are derived for nine sub-samples, narrowly binned in luminosity and redshift. The COSMOS data alone are well fit by a power law, $M_{200} \propto\left(L_{\mathrm{X}}\right)^{\alpha}$, with a slope of $\alpha=0.66 \pm 0.14$. These results significantly extend the dynamic range for which the halo masses of X-ray-selected structures have been measured with weak gravitational lensing. As a result, tight constraints are obtained for the slope of the $M-L_{\mathrm{X}}$ relation. The combination of our group data with previously published cluster data demonstrates that the $M-L_{\mathrm{X}}$ relation is well described by a single power law, $\alpha=0.64 \pm 0.03$, over two decades in mass, $M_{200} \sim 10^{13.5}-10^{15.5} h_{72}^{-1} M_{\odot}$. These results are inconsistent at the $3.7 \sigma$ level with the self-similar prediction of $\alpha=0.75$. We examine the redshift dependence of the $M-L_{X}$ relation and find little evidence for evolution beyond the rate predicted by self-similarity from $z \sim 0.25$ to $z \sim 0.8$.
\end{abstract}

Key words: cosmology: observations - gravitational lensing - large-scale structure of universe

Online-only material: color figures

\footnotetext{
* Based on observations with the NASA/ESA Hubble Space Telescope, obtained at the Space Telescope Science Institute, which is operated by AURA Inc, under NASA contract NAS 5-26555; also based on data collected at the Subaru Telescope, which is operated by the National Astronomical Observatory of Japan; the XMM-Newton, an ESA science mission with instruments and contributions directly funded by ESA Member States and NASA; the European Southern Observatory under Large Program 175.A-0839, Chile; Kitt Peak National Observatory, Cerro Tololo Inter-American Observatory, and the National Optical Astronomy Observatory, which are operated by the Association of Universities for
}

Research in Astronomy, Inc. (AURA) under cooperative agreement with the National Science Foundation; the National Radio Astronomy Observatory which is a facility of the National Science Foundation operated under cooperative agreement by Associated Universities, Inc ; and the Canada-France-Hawaii Telescope with MegaPrime/MegaCam operated as a joint project by the Canada-France-Hawaii-Telescope Corporation, CEA/DAPNIA, the National Research Council of Canada, the Canadian Astronomy Data Centre, the Centre National de la Recherche Scientifique de France, TERAPIX and the University of Hawaii.

${ }^{23}$ Hubble fellow. 


\section{INTRODUCTION}

Groups and clusters of galaxies, formed through the gravitational collapse of massive dark matter halos, are now readily identified up to redshift one and even beyond (e.g., Stanford et al. 2006; Eisenhardt et al. 2008). Baryonic tracers such as redsequence galaxies, typically abundant at the centers of groups and clusters, or X-ray emission from the hot intracluster medium (ICM), have proved to be especially successful in this task (e.g., Gladders \& Yee 2005; Koester et al. 2007; Finoguenov et al. 2007). Nonetheless, these observables only trace the tip of the iceberg given that the vast majority of the underlying mass is in the form of dark matter.

The quantification of the total mass (both dark and baryonic) of groups and clusters of galaxies is an important endeavor from both a cosmological and an astronomical standpoint. In particular, several lines of research would benefit from a clearer understanding of the relationship between the total halo mass of groups and clusters and their baryonic tracers. We outline several briefly here (for a recent review on this subject see Voit 2005). From a cosmological perspective, the number density of groups and clusters as a function of total mass is of fundamental interest because it is sensitive to both the expansion and growth history of the universe and can be used to constrain cosmological parameters such as $\Omega_{m}, \sigma_{8}$, and $\Omega_{\Lambda}$ (e.g., White et al. 1993; Wang \& Steinhardt 1998; Haiman et al. 2001; Rozo et al. 2004; Wang et al. 2004; Bahcall et al. 2004; Rozo et al. 2009). Furthermore, modifications to the laws of gravity, which can be invoked as a possible physical mechanism for acceleration, could imprint telltale signatures in the abundance and dark matter structure of groups and clusters of galaxies (Rapetti et al. 2009; Schmidt et al. 2009). Unfortunately, the common difficulty encountered with each of these enquires is that theories and simulations make darkmatter-based predictions, but our most accessible observables (such as richness or X-ray luminosity) are baryonic in nature.

It has long been recognized that baryonic observables are subject to complex and poorly understood physical processes that make them imperfect dark matter tracers. For example, X-ray studies discovered early on that the theory of pure gravitational collapse which makes simple predictions for the shape and amplitude of X-ray scaling relations (also known as the self-similar model; Kaiser 1986) fail to match observations such as the slope and normalization of the $L_{X}-T$ relation (Voit 2005, and references therein) implying that other non-gravitational (and still much debated) processes have significantly affected the thermodynamic state of the ICM. Additional heating and cooling mechanisms that are invoked to solve this puzzle lead to different predictions for the shape and redshift evolution of X-ray scaling relations. On the one hand, the fact that X-ray scaling relations deviate from simple models is a plague for cosmologists because there is no straightforward recipe to estimate total halo masses. On the other hand, from an astronomical perspective, the comparison between X-ray observables and total halo mass contains valuable clues about the physical processes that govern galaxy formation and the heating and cooling of the ICM.

For all of these reasons, more precise measurements of the mean and scatter in the relationship between total halo mass and various baryonic tracers of groups and clusters of galaxies are highly desirable (e.g., see discussions in Voit 2005; Albrecht et al. 2006).

At present, there are five popular methods for detecting groups and clusters of galaxies: (1) optical detection via the red se- quence (e.g., Gladders \& Yee 2005; Koester et al. 2007); (2) detection via the Sunyaev-Zeldovich (SZ) effect which measures the distortion of the cosmic microwave background spectrum due to the hot ICM (e.g., Sunyaev \& Zeldovich 1970, 1972; Carlstrom et al. 2002; Benson et al. 2004; Staniszewski et al. 2009); (3) detection via X-ray emission (e.g., Böhringer et al. 2000; Hasinger et al. 2001; Finoguenov et al. 2007; Vikhlinin et al. 2009); (4) spectroscopic identification (e.g., Gerke et al. 2005; Miller et al. 2005; Knobel et al. 2009); and (5) detection via weak lensing maps (e.g., Marian \& Bernstein 2006; Miyazaki et al. 2007; Massey et al. 2007b). This last technique is the simplest in terms of the underlying physics and is the only method for which the total halo mass can be directly probed, independently of both the baryons and the dynamical state of the cluster. However, shear maps can only detect the most massive systems $\left(M>10^{14} M_{\odot}\right)$ and are limited to moderate redshifts because the lensing weight function peaks mid way between the source and the observer, with galaxy shapes increasingly difficult to measure at $z>1$. X-ray observations, on the other hand, can more simply probe complete samples of groups and clusters, but departures from virial equilibrium and non-thermal pressure components in the ICM can bias X-ray-based hydrostatic mass estimates (e.g., Nagai et al. 2007). The SZ effect has the attractive property of being redshift independent, and the integrated SZ flux increment, $Y$, may be less sensitive to the baryon physics of cluster cores (Motl et al. 2005; Nagai 2006), but mass measurements with the SZ effect face other challenges such as the identification and removal of radio point sources (Vale \& White 2006), sky confusion owing to projection effects (White et al. 2002), and possibly a larger scatter in the $Y-M$ relation than previously estimated due to feedback processes (Shaw et al. 2008).

Given these considerations, a promising strategy is to employ a robust and efficient cluster detection method (to which the ultimate solution may be a combination of several techniques such as described in Cohn \& White 2009) and to perform an absolute mass calibration of baryonic tracers via weak gravitational lensing (Hoekstra 2007; Rykoff et al. 2008; Bergé et al. 2008).

The focus of this paper is to advance these goals by calibrating the slope and amplitude of the $M-L_{\mathrm{X}}$ relation for galaxy groups using cross-correlation weak lensing in the COSMOS survey (also called "group-galaxy lensing"). Extending weak lensing measurements into the group regime is particularly important in order to extend the dynamic range of weak-lensing-based mass estimates so as to more accurately determine the slopes of scaling relations. Using the COSMOS sample, we show that $\mathrm{X}$-ray detections span a more complete and wide range of redshift and mass than detections via shear maps. Indeed, high redshift and small structures are challenging to detect directly with shear because of the shape of the lensing weight function (see Section 4). Nevertheless, once they have been identified, groups and clusters can be studied via stacking techniques. A notable advantage of this method is that measurements are unaffected by uncorrelated mass along the line of sight, whereas mass estimates for individually detected clusters are subject to 20\% uncertainties (Metzler et al. 2001; Hoekstra 2003; de Putter \& White 2005). The associated drawback with stacking is that the intrinsic scatter around the mean relation is difficult to recover.

In order to employ the stacked weak lensing technique, tight baryonic tracers of halo mass are highly desirable. The $\mathrm{X}$-ray luminosity of groups and clusters is considered to be 
a reasonable tracer of halo mass with a logarithmic scatter in the $M-L_{\mathrm{X}}$ relation of roughly $20 \%-30 \%$ (Stanek et al. 2006; Maughan 2007; Pratt et al. 2009; Rozo et al. 2009; Rykoff et al. 2008; Vikhlinin et al. 2009). A large fraction of this scatter has been shown to be associated with the presence of cool cores and simple excision techniques can reduce the scatter to sub-20\% levels (Maughan 2007; Pratt et al. 2009). Although more tightly correlated mass tracers have been identified such as the $Y_{\mathrm{X}}$ parameter (e.g., Kravtsov et al. 2006)—such indicators require the measurement of an X-ray spectrum which is not possible for most survey data where count rates are low. Our choice of $L_{X}$ as a mass proxy reflects the fact that it is a simple X-ray observable, accessible with survey quality data, and the only one that can be easily measured at high redshift. Temperature measurements may be feasible for a small fraction of high redshift objects but cosmological studies that require complete samples of high redshift groups and clusters will need simple mass proxies like $L_{\mathrm{X}}$. The details of the $M-L_{\mathrm{X}}$ relation are also important (regardless of the choice of a mass proxy) for determining effective volumes as a function of mass in $\mathrm{X}$ ray-flux-limited surveys (Stanek et al. 2006; Vikhlinin et al. 2009).

The layout of this paper is as follows. The data are presented in Section 2, and the theoretical lensing background is developed in Section 3. The construction of the group catalog and the lens selection are specified in Section 4. Details regarding the adopted form of the $M-L_{\mathrm{X}}$ relation are given in Section 5. Our main results are presented in Section 6 followed by our assessment of the systematic errors in Section 7. Finally, we discuss the results and draw up our conclusions in Section 8.

We assume a WMAP5 $\Lambda$ CDM cosmology with $\Omega_{\mathrm{m}}=0.258$, $\Omega_{\Lambda}=0.742, \Omega_{\mathrm{b}} h^{2}=0.02273, n_{\mathrm{s}}=0.963, \sigma_{8}=0.796$, $H_{0}=72 h_{72} \mathrm{~km} \mathrm{~s}^{-1} \mathrm{Mpc}^{-1}$ (Hinshaw et al. 2009). All distances are expressed in physical units of $h_{72}^{-1} \mathrm{Mpc}$. X-ray luminosities are expressed in the $0.1-2.4 \mathrm{keV}$ band, rest frame. The letter $M$ denotes halo mass in general, whereas $M_{200}$ is explicitly defined as $M_{200} \equiv M\left(<r_{200}\right)=200 \rho_{\text {crit }}(z) \frac{4}{3} \pi r_{200}^{3}$, where $r_{200}$ is the radius at which the mean interior density is equal to 200 times the critical density $\left(\rho_{\text {crit }} \equiv 3 H^{2}(z) / 8 \pi G\right)$. The function $E(z) \equiv H(z) / H_{0}=\sqrt{\Omega_{m}(1+z)^{3}+\Omega_{\Lambda}}$ represents the Hubble parameter evolution for a flat metric. All magnitudes are given on the AB system.

\section{THE COSMOS SURVEY}

The COSMOS survey brings together a broad array of panchromatic observations with imaging data from X-ray to radio wavelengths and a large spectroscopic follow-up program (zCOSMOS) with the VLT (Scoville et al. 2007; Koekemoer et al. 2007; Lilly et al. 2007). In particular, the COSMOS program has imaged the largest contiguous area (1.64 degrees ${ }^{2}$ ) to date with the Hubble Space Telescope (HST) using the Advanced Camera for Surveys (ACS) Wide Field Channel (WFC). In addition to the ACS/WFC $\left(I_{F 814 W}\right)$ imaging, the COSMOS field has been targeted by both the XMMNewton (1.5 Ms; Hasinger et al. 2007; Cappelluti et al. 2009) and the Chandra observatories (1.8 Ms, Elvis et al. 2009). The combination of ACS imaging to provide accurate shape measurements, and of XMM-Newton/Chandra imaging, sets the stage for the study of the dark matter halos of galaxy groups via weak lensing techniques. In the following sections, we describe the various data sets and catalogs employed in this analysis.

\subsection{ACS Lensing Data}

Our general scheme for the construction of the COSMOS ACS lensing catalog is based on Leauthaud et al. (2007), and we refer the reader to this publication for details regarding the source extraction and catalog construction-only a brief review will be given here. Since Leauthaud et al. (2007), however, we have made a key improvement regarding the effects of charge transfer inefficiency (CTI) in the ACS CCDs. This aspect in particular is outlined below in greater detail.

In Leauthaud et al. (2007) and Rhodes et al. (2007), we remarked that the COSMOS ACS images are strongly affected by CTI. As charge is transferred during the CCD read-out process, a certain fraction is retained by charge traps (created by cosmic ray hits) in the pixels. This causes flux to be trailed behind objects as the traps gradually release their charge, spuriously elongating them in a coherent direction that mimics a lensing signal. Our previous work employed a parametric scheme to correct for this effect at a catalog level. Although a parametric scheme provides a first-order correction of CTI, it neglects the dependence of the CTI on object size, radial profile, and shape for example. For this reason, in Massey et al. (2009), we have developed a physically motivated correction scheme that operates on the raw data and that has been shown to achieve a $97 \%$ level of correction. Using this scheme, we have produced a new set of raw "unrotated" ACS/WFC data (version 2.0) in which the CTI trailing is reduced by more than an order of magnitude (for further details, see Massey et al. 2009). The raw data are co-added using the same MultiDrizzle set-up as in our previous work.

We use Version 2.5.0 of the SExtractor photometry package (Bertin \& Arnouts 1996) to extract a source catalog of positions from the v2.0 ACS data using the same "Hot-Cold" method as in Leauthaud et al. (2007). Defects and diffraction spikes are carefully removed from the catalog, leaving a total of $\sim 1.2 \times 10^{6}$ objects to a limiting magnitude of $I_{F 814 W}=26.5$.

The next step is to measure the shapes of galaxies and to correct them for the distortion induced by the time varying ACS point-spread function (PSF) as described in Rhodes et al. (2007). As compared to Rhodes et al. (2007), the parametric CTI correction is no longer applied because this effect has already been removed in the raw images.

Simulated images are used to derive the shear susceptibility factors that are necessary in order to transform shape measurements into unbiased shear estimators. Finally, for every galaxy, we derive a shape measurement error and utilize this quantity to extract the intrinsic shape noise of the galaxy sample. Representing a number density of 66 galaxies arcminute ${ }^{-2}$, the final COSMOS weak lensing catalog contains $3.9 \times 10^{5}$ galaxies with accurate shape measurements.

\subsection{Photometric and Spectroscopic Redshifts}

Redshift information is critical for both the lens and source populations because it allows one to correctly scale lensing relations to remove foreground contamination and to study weak lensing signals in terms of physical instead of angular distances. We use an updated and improved version of the photometric redshifts (hereafter photozs) presented in Ilbert et al. (2009) which have been computed with over 30 bands of multi-wavelength data. The main differences between the Ilbert et al. (2009) catalog and the one that we use here are the addition of deep $H$-band data and small improvements in the template-fitting technique. Details regarding the data and 
the photometry can be found in Capak et al. (2007; see also McCracken et al. 2009).

Photozs were estimated using a $\chi^{2}$-template-fitting method (Le Phare) and compared with large spectroscopic samples from the Very Large Telescope (VLT) Visible Multi-Object Spectrograph (VIMOS; Lilly et al. 2007) and the Keck Deep Extragalactic Imaging Multi-Object Spectrograph (DEIMOS). The combined spectroscopic redshift sample comprises: 10,801 galaxies at $z \sim 0.48,696$ at $z \sim 0.74$, and 870 at $z \sim 2.2$. The dispersion in the photozs as measured by comparing to the spectroscopic sample is $\sigma_{\Delta z /(1+\mathrm{zspec})}=0.007$ at $i_{A B}^{+}<22.5$, where $\Delta z=z_{\text {spec }}-z_{\text {phot }}$. The deep IR and Infrared Array Camera (IRAC) data enable the photozs to be calculated even at fainter magnitudes with a reasonable accuracy of $\sigma_{\Delta z /(1+\mathrm{zspec})}=$ 0.06 at $i_{A B}^{+} \sim 24$. In particular, deep $J, H, K$, and $u^{*}$ band data allow for a better estimation of the photozs at $z>1$ via the $4000 \AA$ break which is shifted into the infrared (IR).

Larger samples of spectroscopic redshifts at $z>1$ will ultimately be required to define the most trustworthy magnitude and redshift range for the source galaxies (in a similar fashion to Mandelbaum et al. 2008). Meanwhile, to mitigate the effects of photoz uncertainties, we use a conservative source galaxy selection which will be discussed in more detail in Section 7. In short, we reject all source galaxies with a secondary peak in the redshift probability distribution function (i.e., the parameter ZP_SEC is non-zero in the Ilbert et al. 2009 catalog). This cut is aimed to reduce the number of catastrophic errors (a preferential shift in a certain population of galaxies from one redshift bin to another) in the source catalog. The ZP_SEC $>0$ galaxy population is expected to contain a large fraction of catastrophic errors (roughly 40\%-50\%, Ilbert et al. 2006, 2009). The photoz quality cuts reduce the number density of source galaxies from 66 to 34 galaxies arcminute ${ }^{-2}$. The final mean redshift and magnitude of the source sample are $\langle z\rangle \sim 1$ and $\left\langle I_{F 814 W}\right\rangle \sim 24$. In addition to these quality cuts, for each lens-source pair, we demand that $z_{\text {source }}-z_{\text {lens }}>\sigma_{68 \%}\left(z_{\text {source }}\right)$ and that $z_{\text {source }}-z_{\text {lens }}>0.1$ to ensure a clean selection of background galaxies.

\subsection{Stellar Mass Estimates}

Stellar masses are used to identify the Most Massive Central Galaxy (MMCG; see Section 4.4 for more details) and are estimated using the Bayesian code described in Bundy (2006a, 2006b). Briefly, an observed galaxy's spectral energy distribution (SED) and photoz are referenced to a grid of models constructed using the Bruzual \& Charlot (2003) synthesis code. The grid includes models that vary in age, star formation history, dust content, and metallicity. At each grid point, the probability that the observed SED fits the model is calculated, and the corresponding stellar mass to $K$-band luminosity ratio and stellar mass are stored. By marginalizing over all parameters in the grid, the stellar mass probability distribution is obtained. The median of this distribution is taken as the stellar mass estimate, and the width encodes the uncertainty due to degeneracies and uncertainties in the model parameter space. The final uncertainty on the stellar mass also includes the $K$-band photometry uncertainty as well as the expected error on the luminosity distance that results from the photoz uncertainty. The typical final uncertainty is $0.2-0.3$ dex.

\subsection{XMM and Chandra Data}

The entire COSMOS region has been mapped through 54 overlapping XMM-Newton pointings, and additional Chandra observations cover the central region $\left(0.9\right.$ degrees $\left.^{2}\right)$ to higher resolution. A composite XMM-Newton and Chandra mosaic has been used to detect and measure the fluxes of groups and clusters to a $4 \sigma$ detection limit ${ }^{24}$ of $1.0 \times 10^{-15} \mathrm{erg} \mathrm{cm}^{-2} \mathrm{~s}^{-1}$ over $96 \%$ of the ACS field. The general data reduction process can be found in Finoguenov et al. (2007), and details regarding improvements and modifications to the original catalog are given in Section 4. In particular, the group and cluster catalog used in this paper features a more conservative point-source removal procedure than in Finoguenov et al. (2007). Redshift identification has also improved thanks to the increased photoz accuracy and to the availability of more spectroscopic data (see Section 4.3). In total, the catalog used in this paper contains $206 \mathrm{X}$-ray groups and clusters of galaxies over 1.64 degrees $^{2}$, spanning the redshift range $0<z<1.6$ and with a rest-frame $0.1-2.4 \mathrm{keV}$ luminosity range between $10^{41}$ and $10^{44} \mathrm{erg} \mathrm{s}^{-1}$.

\section{THEORETICAL LENSING FRAMEWORK}

\subsection{From Galaxy Shapes to $\Delta \Sigma$}

In the weak gravitational lensing limit, the observed shape $\varepsilon_{\text {obs }}$ of a source galaxy is directly related to the lensing induced shear $\gamma$ according to

$$
\varepsilon_{\mathrm{obs}}=\varepsilon_{\mathrm{int}}+\gamma
$$

where $\varepsilon_{\text {int }}$ is the source galaxy's intrinsic shape that would be observed in the absence of gravitational lensing. In our notation, $\varepsilon_{\text {int }}, \varepsilon_{\text {obs }}$, and $\gamma$ are spin-2 tensors. The above relationship indicates that galaxies would be ideal tracers of the distortions caused by gravitational lensing if the intrinsic shape $\varepsilon_{\text {int }}$ of each source galaxy was known a priori. However, lensing measurements exhibit an intrinsic limitation, encoded in the width of the ellipticity distribution of the galaxy population, noted here as $\sigma_{\text {int }}$, and often referred to as the "intrinsic shape noise." Because the intrinsic shape noise (of order $\sigma_{\text {int }} \sim 0.27$; Leauthaud et al. 2007) is significantly larger than $\gamma$ (typically $\gamma \sim 0.05$ for this work), shears must be estimated by averaging over a large number of source galaxies.

Throughout this paper, the gravitational shear is noted as $\gamma$, whereas $\tilde{\gamma}$ represents our estimator of $\gamma$. The uncertainty in the shear estimator is a combination of unavoidable intrinsic shape noise, $\sigma_{\text {int }}=\sqrt{\left\langle\varepsilon_{\text {int }}^{2}\right\rangle}$, and of shape measurement error, $\sigma_{\text {meas }}$ :

$$
\sigma_{\tilde{\gamma}}^{2}=\sigma_{\text {int }}^{2}+\sigma_{\text {meas }}^{2}
$$

We will refer to $\sigma_{\tilde{\gamma}}$ as "shape noise," whereas $\sigma_{\text {int }}$ will be called the "intrinsic shape noise." The former includes the shape measurement error and will vary according to each specific data set and shape measurement method. Averaged over the whole COSMOS field, the weak lensing distortions represent a negligible perturbation to Equation (2). The intrinsic shape noise and measurement error for COSMOS have been characterized in Leauthaud et al. (2007) by using a sample of 27,000 source galaxies that lie within the overlapping regions of adjacent pointings. The shape measurement error is determined for every source galaxy as a function of size and magnitude. For this paper, an intrinsic shape noise of $\sigma_{\text {int }}=0.27$ is assumed.

The derivation of our shear estimator is presented in Leauthaud et al. (2007). We employ the RRG method (see Rhodes et al. 2000, for further details) for galaxy shape measurements. Briefly, we form $\tilde{\gamma}$ from the PSF-corrected ellipticity

\footnotetext{
24 Quoted detection limits correspond to the wavelet scalewise reconstruction. See Finoguenov et al. (2007) for more details.
} 
Table 1

Various Properties for Each of the Nine Bins

\begin{tabular}{|c|c|c|c|c|c|c|c|c|c|c|}
\hline Bin ID & $N_{\text {LENS }}$ & $\begin{array}{c}\left\langle L_{X} \cdot E(z)^{-1}\right\rangle \\
\left(10^{42} h_{72}^{-2} \operatorname{erg~s}^{-1}\right) \\
\end{array}$ & $f_{\mathrm{CC}}{ }^{\mathrm{a}}$ & $\begin{array}{c}\left\langle M_{200}\right\rangle \\
\left(10^{13} h_{72}^{-1} M_{\odot}\right)\end{array}$ & $\langle z\rangle$ & $\mathrm{E}(\langle z\rangle)$ & $\begin{array}{c}L_{Z} \\
\left(10^{-4} h_{72}^{-2} \mathrm{pc}^{2} \mathrm{M}_{\odot}^{-2}\right)\end{array}$ & $f_{\text {bias }}$ & $f_{\text {boost }}$ & $f_{\text {bias }} \times f_{\text {boost }}$ \\
\hline $\mathrm{A} 0$ & 1 & $31.14 \pm 0.49$ & 1.0 & $14.9_{-4.8}^{+7.1}$ & 0.22 & 1.10 & 3.16 & 1.01 & 1.00 & 1.01 \\
\hline A1 & 3 & $13.75 \pm 0.50$ & 1.0 & $8.2_{-2.3}^{+3.3}$ & 0.36 & 1.18 & 3.78 & 1.02 & 1.00 & 1.03 \\
\hline A 2 & 3 & $6.04 \pm 0.28$ & 1.17 & $9.9_{-2.5}^{+3.3}$ & 0.35 & 1.17 & 3.77 & 1.01 & 1.00 & 1.02 \\
\hline A3 & 11 & $2.21 \pm 0.15$ & 1.08 & $3.2_{-0.9}^{+1.3}$ & 0.36 & 1.18 & 3.77 & 1.02 & 1.00 & 1.02 \\
\hline A4 & 7 & $0.90 \pm 0.06$ & 1.03 & $2.1_{-0.8}^{+1.5}$ & 0.23 & 1.11 & 3.20 & 1.01 & 1.00 & 1.01 \\
\hline A5 & 13 & $1.24 \pm 0.09$ & 1.05 & $1.1_{-0.4}^{+0.8}$ & 0.35 & 1.18 & 3.77 & 1.01 & 1.00 & 1.02 \\
\hline A6 & 11 & $3.65 \pm 0.21$ & 1.17 & $3.3_{-1.1}^{+1.7}$ & 0.50 & 1.27 & 3.96 & 1.03 & 1.01 & 1.04 \\
\hline A7 & 23 & $4.72 \pm 0.24$ & 1.13 & $2.6_{-0.7}^{+1.1}$ & 0.69 & 1.41 & 3.84 & 1.04 & 1.02 & 1.06 \\
\hline A8 & 21 & $10.51 \pm 0.50$ & 1.14 & $7.6_{-1.8}^{+2.3}$ & 0.90 & 1.58 & 3.65 & 1.03 & 1.05 & 1.08 \\
\hline
\end{tabular}

Note. ${ }^{\text {a }}$ The cool-core correction factor that is applied to Column 3 (see description in Section 2.1).

according to

$$
\tilde{\gamma}=C \times \frac{\varepsilon_{\mathrm{obs}}}{G}
$$

where the shear susceptibility factor ${ }^{25}, G$, is measured from moments of the global distribution of $\varepsilon_{\mathrm{obs}}$ and other, higher order shape parameters (see Equation (28) in Rhodes et al. 2000). Using a set of simulated images similar to those of Shear TEsting Program (STEP; Heymans et al. 2006; Massey et al. 2007a) but tailored exclusively to this data set, we find that, in order to correctly measure the input shear on COSMOS-like images, the RRG method requires an overall calibration factor of $C=\left(0.86_{-0.05}^{+0.07}\right)^{-1}$ (see Leauthaud et al. 2007, for more details).

The shear signal induced by a given foreground mass distribution on a background source galaxy will depend on the transverse proper distance between the lens and the source and on the redshift configuration of the lens-source system. A lens with a projected surface mass density, $\Sigma(r)$, will create a shear that is proportional to the surface mass density contrast, $\Delta \Sigma(r)$ :

$$
\Delta \Sigma(r) \equiv \bar{\Sigma}(<r)-\bar{\Sigma}(r)=\Sigma_{\text {crit }} \times \gamma_{t}(r) .
$$

Here, $\bar{\Sigma}(<r)$ is the mean surface density within the proper radius $r, \bar{\Sigma}(r)$ is the azimuthally averaged surface density at radius $r$ (e.g., Miralda-Escude 1991; Wilson et al. 2001), and $\gamma_{t}$ is the tangentially projected shear. The geometry of the lens-source system intervenes through the critical surface mass density, $\Sigma_{\text {crit }}$, which depends on the angular diameter distances to the lens $\left(D_{\mathrm{OL}}\right)$, to the source $\left(D_{\mathrm{OS}}\right)$, and between the lens and source $\left(D_{\mathrm{LS}}\right)$ :

$$
\Sigma_{\text {crit }}=\frac{c^{2}}{4 \pi G_{\mathrm{N}}} \frac{D_{\mathrm{OS}}}{D_{\mathrm{OL}} D_{\mathrm{LS}}},
$$

where $G_{\mathrm{N}}$ represents Newton's constant. Hence, if redshift information is available for every lens-source pair, each estimate of $\gamma_{t}$ can be directly converted to an estimate of $\Delta \Sigma$ which is a more desirable quantity because it depends only on the mass distribution of the lens.

To measure $\Delta \Sigma(r)$ with high signal to noise, the lensing signal must be stacked over many foreground lenses and background sources. For every $i$ th lens and $j$ th source separated by a proper distance $r_{i j}$, an estimator of the mean excess projected surface mass density at $r_{i j}$ is computed according to

$$
\Delta \tilde{\Sigma}_{i j}\left(r_{i j}\right)=\tilde{\gamma}_{t, i j} \times \Sigma_{\mathrm{crit}, i j}
$$

\footnotetext{
${ }^{25}$ Not to be confused with Newton's constant which we have noted as $G_{\mathrm{N}}$.
}

where $\tilde{\gamma}_{t, i j}$ is the tangential shear of the source relative to the lens. The COSMOS photometric redshifts described in Section 2.2 are used to estimate $\Sigma_{\text {crit }, i j}$ for every lens-source pair. In order to optimize the signal to noise, an inverse variance weighting scheme is employed when $\Delta \Sigma_{i j}$ is summed over many lens-source pairs. Each lens-source pair is attributed a weight that is equal to the estimated variance of the measurement:

$$
w_{i j}=\frac{1}{\left(\Sigma_{\mathrm{crit}, i j} \times \sigma_{\tilde{\gamma}, i j}\right)^{2}} .
$$

In this manner, faint small galaxies which have large measurement errors are downweighted with respect to sources that have well-measured shapes.

In general, for the types of lenses studied in this paper (groups and low mass clusters of galaxies), the signal to noise per lens is not high enough to measure $\Delta \Sigma$ on an object by object basis so instead we stack the signal over many lenses. For a given sample of lenses, the total excess projected surface mass density is the weighted sum over all lens-source pairs:

$$
\Delta \Sigma=\frac{\sum_{j=1}^{N_{\text {Lens }}} \sum_{i=1}^{N_{\text {Source }}} w_{i j} \times \tilde{\gamma}_{t, i j} \times \Sigma_{\text {crit }, i j}}{\sum_{j=1}^{N_{\text {Lens }}} \sum_{i=1}^{N_{\text {Source }}} w_{i j}} .
$$

\subsection{Non-weak Shear}

Equation (1) and subsequent derivations only hold in the weak gravitational lensing limit, that is to say when $\gamma \ll 1$ and $\kappa \ll 1$ $\left(\kappa=\Sigma / \Sigma_{\text {crit }}\right.$ is the convergence). In reality, galaxy shapes trace the reduced shear, $g=\gamma /(1-\kappa)$. The masses of the groups that we are probing are such that the weak lensing assumption can begin to break down in the most inner bins $\left(r<100 h_{72}^{-1} \mathrm{kpc}\right)$ and for high redshift source galaxies. In this regime, $g \sim \gamma$ is no longer a valid assumption. Following the methodology outlined in Johnston et al. (2007) and Mandelbaum et al. (2006a), it can be shown that our weighted estimator for $\Delta \Sigma$ will have a second-order contribution:

$$
\begin{gathered}
\Delta \tilde{\Sigma}=\Delta \Sigma+\Delta \Sigma \Sigma L_{\mathrm{Z}}, \\
L_{\mathrm{Z}}=\frac{\left\langle\Sigma_{\text {crit }}^{-3}\right\rangle}{\left\langle\Sigma_{\text {crit }}^{-1}\right\rangle} .
\end{gathered}
$$

For further details, see Equation (19) in Johnston et al. (2007) and Appendix A in Mandelbaum et al. (2006a). In a similar fashion to Johnston et al. (2007), we ignore the variations of 
$L_{Z}$ within various radial bins. However, because our lens sample spans a large redshift range, we do not use a constant value of $L_{Z}$ for all redshift bins. Instead, $L_{Z}$ is calculated from the data for each redshift bin. Our values for $L_{\mathrm{Z}}$ are given in Table 1.

\subsection{A Model for Predicting $\Delta \Sigma$}

A halo model approach is used to model the surface mass density contrast $\Delta \Sigma$ as a function of transverse separation (e.g., Mandelbaum et al. 2005, 2006a, 2006b; Yoo et al. 2006; Johnston et al. 2007). The total signal is modeled as the sum of two distinct components that dominate the signal at different scales. The first term is due to the baryonic mass contained within the central galaxy (CG) and only contributes at scales below $\sim 50 \mathrm{kpc}$. The second term dominates the signal on intermediate to large scales $(\sim 50 \mathrm{kpc}$ to a few Mpc) and represents the group-scale dark matter halo (also known as the "one-halo term"). On the largest scales (above several Mpc), the clustering of halos among themselves produces a contribution to $\Delta \Sigma$ via the so-called "two-halo term." However, we have found that the two-halo term is mostly sub-dominant at the scales that we probe. We have tested that the exclusion of the two-halo term has no impact on the results of this study and we therefore neglect this term hereafter.

The baryonic mass of the CGs can have a non-negligible contribution to $\Delta \Sigma$ at small transverse separations from the center of the stacked ensemble. Although the baryons typically follow a Sersic profile, at the scales of interest for this study, well above a few effective radii $(>40 \mathrm{kpc})$, the lensing contribution of the baryons can be modeled by a simple point source scaled to $\left\langle M_{\mathrm{CG}}\right\rangle$ the average stellar mass of the CGs (also see Sections 2.3 and 4.4):

$$
\Delta \Sigma_{\text {stellar }}(r)=\frac{\left\langle M_{\mathrm{CG}}\right\rangle}{\pi r^{2}} .
$$

To be more precise, the baryons that have not yet transformed into stars should also be considered. However, the majority of non-stellar group baryons are in the form of diffuse hot gas spread throughout the halo, in rough equilibrium with the dark matter potential. To first order, the gas mass contribution should follow the dark matter distribution.

We assume that the density profiles of dark matter halos follow Navarro-Frenk-White (NFW) profiles (Navarro et al. 1997). In this work, halo mass is defined as $M_{200} \equiv$ $M\left(<r_{200}\right)=200 \rho_{\text {crit }}(z) \frac{4}{3} \pi r_{200}^{3}$, and $C_{200}$ denotes the halo concentration. Numerous studies have demonstrated that the mass, concentration, and characteristic formation epoch of dark matter halos are closely linked and on average, smaller halos tend to have higher concentrations (Bullock et al. 2001; Wechsler et al. 2002; Macciò et al. 2007; Zhao et al. 2009). For this study, we adopt the Zhao et al. (2009) ${ }^{26}$ mass-concentration relation for a WMAP5 cosmology. By adopting this relation, $\Delta \Sigma_{\text {nfw }}$ is fully described by two parameters, namely $M_{200}$ and redshift. Analytical formulas for the $\Delta \Sigma$ corresponding to a NFW profile can be found in Wright \& Brainerd (2000) and in Bartelmann (1996).

The additional gravitational potential due to the baryons is expected to modify the density profiles of dark matter halos via adiabatic contraction (Gnedin 2004; Sellwood \& McGaugh 2005). Nevertheless, Mandelbaum et al. (2006a) have shown that this has a negligible effect on the lensing signal on the scales that we consider (above $40 \mathrm{kpc}$ ), and we neglect this effect for this work.

\footnotetext{
26 See http://www.shao.ac.cn/dhzhao/mandc.html.
}

The final model that we use for the weighted estimate $\Delta \tilde{\Sigma}$ is

$$
\Delta \tilde{\Sigma}=\Delta \Sigma_{\text {stellar }}+\Delta \Sigma_{\mathrm{nfw}}+\Delta \Sigma_{\mathrm{nfw}} \Sigma_{\mathrm{nfw}} L_{\mathrm{Z}}
$$

We have included the second-order contribution to $\Delta \tilde{\Sigma}$ from non-weak shear. Note that only the dark matter halo contributes to this term because $\Sigma_{\text {stellar }}$ is zero at the scales that we probe.

\section{X-RAY GROUP SELECTION}

\subsection{X-ray Selection versus Shear Maps}

Among group and cluster probes, X-rays are perhaps the cleanest and the most complete selection method. First, $\mathrm{X}$-ray emission depends on the square of the gas density and so X-rays pick up the cores of dense structures more accurately than SZ and are less prone to projection effects. Second, unlike optical techniques which rely on galaxy properties, X-rays yield a complete sample of groups and clusters, irrespective of their galaxy content. Finally, X-rays probe a wider range in mass and redshift than shear maps which are fundamentally limited by the shape of the lensing weight function. To illustrate the magnitude of this effect, in the upper panel of Figure 1 we show the expected lensing detection significance of X-ray structures in COSMOS as a function of mass and redshift. The lower panel in Figure 1 shows the comoving volume probed by the survey per unit redshift. The theoretical lensing detection significance is derived according to the method outlined in Hamana et al. (2004) assuming a smoothed COSMOS redshift distribution and isolated NFW profiles truncated at the virial radius. A source density of 66 galaxies $\mathrm{arcmin}^{-2}$ and an average shape noise of $\sigma_{\tilde{\gamma}}=0.31$ are assumed. Lensing $\mathrm{S} / \mathrm{N}$ curves are based on fixed-scale Gaussian smoothing with a one arcminute smoothing kernel (an optimal filter would pick up slightly more signal). Figure 1 demonstrates that lensing alone cannot detect low mass or high redshift objects. Instead one must resort to other detection techniques such as X-rays. Note that although the low redshift lensing sensitivity is relatively good, the volume probed is also quite limited. It is also important to note that the depth of the COSMOS data will probably exceed any near-future space-based mission ${ }^{27}$. In this respect, Figure 1 can be considered as a realistic upper limit for lensingbased structure detection in the near future (for both ground and space-based observatories).

\subsection{Construction of the Group Catalog}

The group catalog used for this work is an improved version of the catalog presented in Finoguenov et al. (2007, hereafter F07) obtained by using additional XMM-Newton and Chandra data and by applying a new procedure for the removal of point sources (A. Finoguenov et al. 2010, in preparation). The group catalog contains a total of 206 systems in the COSMOS ACS field, 170 of which are at $z<1$.

The group catalog is constructed from both the XMM-Newton and the Chandra mosaics. All features with spatial extents below $16^{\prime \prime}$ are removed before co-adding the two mosaics. The combined mosaic is used to search for sources with spatial extents on both $32^{\prime \prime}$ and 64" scales using the wavelet decomposition technique described in Vikhlinin et al. (1998). Both Chandra and XMM-Newton have a spatial resolution that is better than $30^{\prime \prime}$. The full width half-maximum (FWHM) of

\footnotetext{
27 The fiducial depth of EUCLID and JDEM is $I \sim 25.5$.
} 


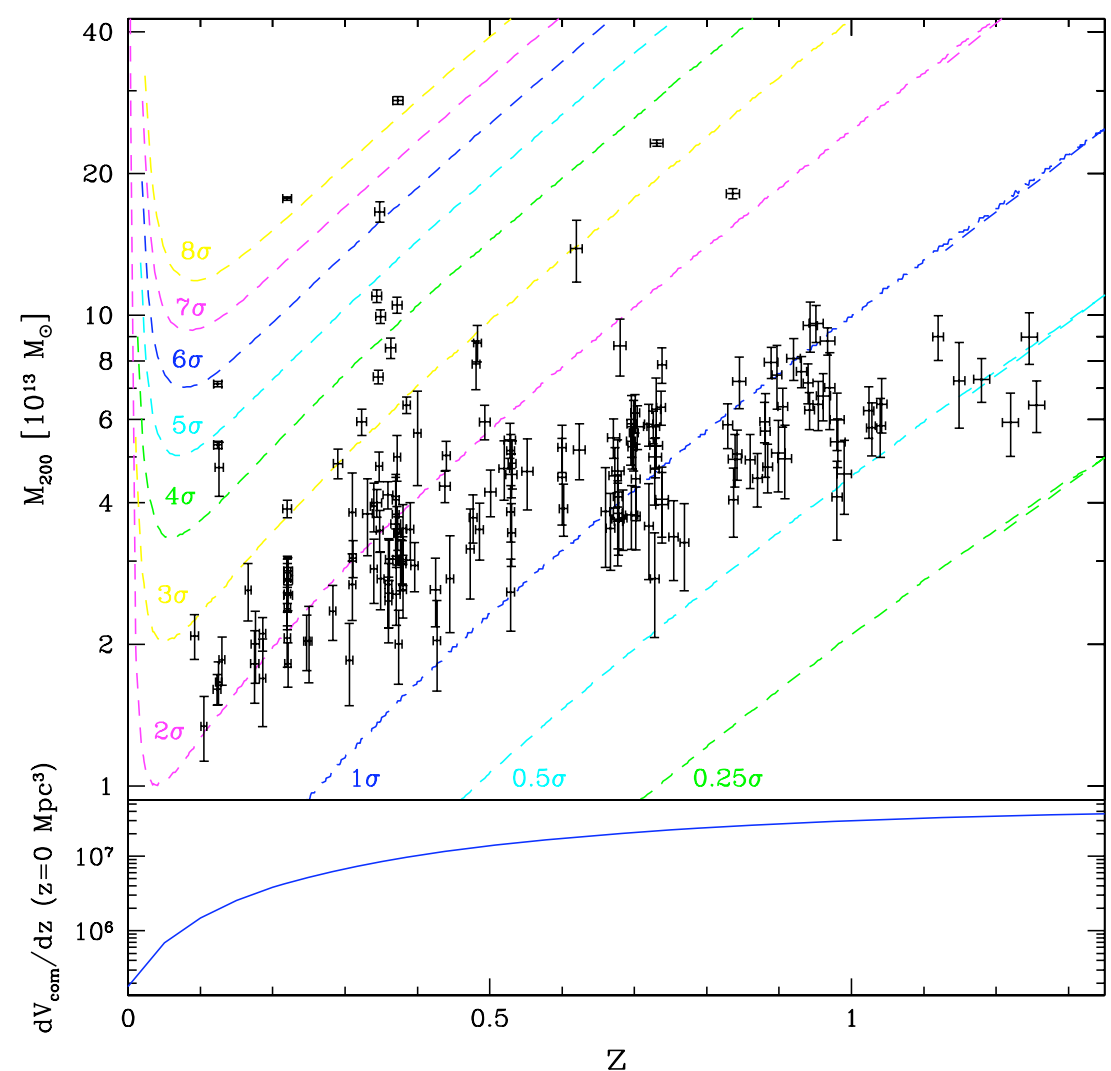

Figure 1. Upper panel: theoretically computed lensing detection significance (dashed curves) of X-ray structures (data points) in the COSMOS field as a function of mass and redshift. Lensing detection significance values have been derived with the method outlined in Hamana et al. (2004) assuming a smoothed COSMOS redshift distribution and a source density of 66 galaxies arcminute ${ }^{-2}$. The predictions in this figure represent a realistic upper limit for all near-future weak-lensing surveys, including space-based missions such as JDEM and EUCLID. Indeed, the survey depth of these missions is unlikely to exceed the current depth of COSMOS. Direct structure detection via shear maps is limited by the lensing weight function at high redshifts and at low masses. To identify such structures, one must resort to other detection techniques. Lower panel: comoving volume probed by the $\left(1.64\right.$ degrees $\left.{ }^{2}\right)$ survey per unit redshift. Although the low redshift lensing sensitivity is relatively good, the volume probed is also quite limited.

(A color version of this figure is available in the online journal.)

the PSF is approximately equal to $16^{\prime \prime}$ for XMM-Newton, and varies between $3^{\prime \prime}$ and $8^{\prime \prime}$ for Chandra.

Total X-ray fluxes are obtained from the measured fluxes by assuming a beta profile and by removing the flux that is due to embedded active galactic nuclei (AGNs) point sources. Previous surveys have often assumed that all of the X-ray fluxes within an extended source area are due to cluster emission (e.g., Böhringer et al. 2004). However, this assumption breaks down for deep surveys such as COSMOS. In particular, we have calculated that on average, AGNs contribute up to $30 \%$ of the extended $\mathrm{X}$-ray emission in the $0.5-2 \mathrm{keV}$ band, while for ROSAT All Sky Survey (RASS) clusters, the estimated value is less than $2 \%$. Point-source removal is thus necessary for COSMOS. However, the procedure that removes the flux from point sources also removes flux from cool cores and as a consequence, the total flux is underestimated. For comparison with other work, it is important to correct for this accidental removal of the flux from cool cores. There have been claims that the cool-core fraction is evolving with redshift (Jeltema et al. 2005; Vikhlinin et al. 2007; Maughan 2007). We have therefore developed a method to correct for this effect directly from the data by using the highresolution Chandra observations of the COSMOS field which cover a contiguous area of 0.5 degrees $^{2}$ with the best PSF ( $3^{\prime \prime}$, Elvis et al. 2009), sufficient to distinguish between cool cores and AGNs. Taking advantage of these data, we compute a coolcore correction factor (noted $f_{\mathrm{CC}}$ ) using the following procedure. To begin with, groups inside the high-resolution Chandra area are binned into the same nine bins as used for the lensing analysis (see Section 6.1). Next, wavelet scales below 4" are used to remove point sources. Finally, the Chandra flux is stacked in each of the nine bins, and a $16^{\prime \prime}$ aperture is used to estimate the average cool-core flux. The results are listed in Table 1 and range from a $3 \%$ to a $17 \%$ flux correction.

Rest-frame luminosities are calculated from the total flux following $L_{0.1-2.4 \mathrm{keV}}=4 \pi d_{L}^{2} K(z, T) C_{\beta}(z, T) F_{\mathrm{d}}$, where $K(z, T)$ is the $K$-correction, and $C_{\beta}(z, T)$ is an iterative correction factor (see F07). The uncertainty in $K(z, T)$ affects groups with luminosities below $10^{42} h_{72}^{-2} \mathrm{erg} \mathrm{s}^{-1}$ and so only concerns a few of the systems considered here. For the $L_{\mathrm{X}}-T$ relation, we have used $k T / k e V=0.2+6 \times 10^{\left.\left(\log \left(L_{X} E(z)^{-1}\right)-44.45\right) / 2.1\right)}$, which introduces a break at group scales (as discussed in Voit 2005) but reproduces the Markevitch (1998) result at cluster scales. Recent work by Pratt et al. (2009) has derived a similar slope for the $L_{X}-T$ relation for clusters as Markevitch (1998). The behavior of the $L_{X}-T$ relation is not very well established at low temperatures; however, exploring the effects of a different $L_{X}-T$ relations is beyond the scope of this paper and is left for future work.

Quality flags (named hereafter "XFLAG") are derived for the entire group catalog based on visual inspection. ${ }^{28}$ XFLAG $=$ 1 is assigned to groups with a single optical counterpart and with a clear X-ray peak. These are mostly highly significant

\footnotetext{
${ }^{28}$ Visual inspection is performed on the XMM-Newton, ACS, and Subaru data simultaneously.
} 
X-ray detections (over $6 \sigma$ ) in zones free of projection effects. The flag XFLAG $=2$ is assigned to systems for which the extended X-ray emission is subject to projection effects but for which the various projections can be disentangled. Systems with questionable optical/IR counterparts are assigned $\mathrm{XFLAG}=3$. These are primarily high $-z(z>1)$ candidates that are not considered in this work. XFLAG $=4$ indicates that there are several equally possible optical counterparts and that the $\mathrm{X}$-ray flux cannot be disentangled for projection effects. $\mathrm{XFLAG}=5$ is assigned to systems for which the optical counterpart is uncertain and XFLAG $=6$ to identified extended emission not associated with galaxy groups (these are mainly interacting galaxies and X-ray jets, V. Smolcic et al. 2010, in preparation). XFLAG is assigned to unidentified emission, and XFLAG $=8$ assigned to systems located in the masked-out regions (edges of the survey and regions near bright stars). In this work, we only consider systems with XFLAG $=1$ or XFLAG $=2$.

To ensure a high-quality and robust lens catalog, in addition to the X-ray quality flags, all systems have been visually inspected and flagged for proximity to the edge of the ACS field, and contamination by the presence of a bright foreground star which will affect both the lensing measurements and the determination of the central group galaxy. All systems with such flags were removed from the lens catalog. In total, after all quality cuts, the lens catalog contains 127 systems at $z \leqslant 1$. None of these quality cuts are expected to bias the remaining sample but will improve the estimations of both $L_{\mathrm{X}}$ and $\mathrm{M}_{200}$.

\subsection{Redshift Determination}

The optical counterparts of X-ray sources are identified using a sophisticated red-sequence method. The details of this technique are presented in Finoguenov et al. (2009)—only a brief outline is given here. Along the line of sight of each X-ray source, the redshift range $0<z<2.5$ is probed for potential red-sequence overdensities. For a given redshift $z=z_{\mathrm{RS}}$, with $0<z_{\mathrm{RS}}<2.5$, galaxies are selected within an aperture of $0.5 \mathrm{Mpc}$ (physical) from the center of the X-ray emission such that $\left|z_{\text {phot }}-z_{\mathrm{RS}}\right|<0.2$. The apparent size of the aperture is defined in terms of a physical scale and will vary with $z_{\mathrm{RS}}$. Weights are derived for all galaxies according to their proximity to the center of the X-ray emission and to the comparison of both their color and magnitude to a model red sequence at $z_{\mathrm{RS}}$. The red-sequence detection significance is determined by applying the same procedure to random COSMOS fields. If there are multiple red-sequence overdensities along the line of sight, the one with the highest significance is selected. Red-sequence redshifts are then refined using spectroscopic information whenever possible.

Of the 127 systems at $z \leqslant 1$ (after the quality cuts), $81 \%$ have two or more spectroscopically confirmed members, $7 \%$ have one spectroscopically confirmed member, and $12 \%$ have a redshift that is determined solely by the red-sequence method. The average redshift error for the group ensemble at $z \leqslant 1$ is estimated at 0.006 , somewhat larger than the typical velocity dispersion of our groups which is about $300 \mathrm{~km} \mathrm{~s}^{-1}$.

\subsection{Center Determination}

Stacked weak lensing measurements require the identification of the dark matter density peak. Centroid errors will lead to a smoothing of the lensing signal on small scales and to an underestimate of halo mass (e.g., see discussion in Johnston et al. 2007). With the exception of on-going, nearly equal mass ratio mergers, the centroid of the $\mathrm{X}$-ray emission should indicate where the potential well is deepest. Halo centers are also often assumed to contain CGs which can be used as good tracers on condition that they can be correctly identified.

The wavelet-reconstructed X-ray image is analyzed with SExtractor (Bertin \& Arnouts 1996) to determine the centers and two-dimensional shapes of the extended X-ray emission. The accuracy of the determination of the X-ray center is higher for XFLAG $=1$ systems than for XFLAG $=2$ systems which are somewhat affected by projection effects. The maximum uncertainty of the peak for XFLAG $=2$ systems is determined by the size of the wavelet scale, which is $32^{\prime \prime}$. Although the $\mathrm{X}$-ray centroid is not precise enough to be used directly in most cases (of 127 system that remain after the quality cuts described in previous sections, 55 have XFLAG $=1$ and 72 have XFLAG $=2$ ), it is precise enough to be used as a strong prior on the location of the CG. Indeed, the maximum uncertainty in the X-ray centroid is $32^{\prime \prime}\left(193 h_{72}^{-1} \mathrm{kpc}\right.$ at $\left.z=0.5\right)$; however, most systems have a smaller positional uncertainty than this. For comparison purposes, the average projected radial offset for misidentified CGs in MaxBCG is larger than $600 h_{72}^{-1} \mathrm{kpc}$ (Figure 5, Johnston et al. 2007), note that their distances must be converted to our assumed value of $H_{0}$.

In many previous studies, Brightest Cluster Galaxies (BCGs) have been associated with the CGs of group and cluster halos. Given the ambiguity in the choice of the filter in which BCG galaxies should be taken as "brightest" as well as the sensitivity of optical luminosity to recent star formation, we prefer the utilization of the Most Massive Central Galaxy (MMCG) located near the peak X-ray emission (where massive refers to stellar mass). We assume that the MMCG can be used to trace the center of the dark matter halos of groups and clusters. An automatic algorithm was developed to identify MMCGs. Briefly, for each X-ray group, a broad group member selection is made by selecting galaxies within $800 \mathrm{kpc}$ of the peak X-ray emission such that $\left|z_{\text {phot }}-z_{\text {group }}\right|<0.03 \times\left(1+z_{\text {group }}\right)$. Next, galaxies are rank-ordered by their stellar mass and weighted by the proximity to the peak X-ray emission-the MMCG is the galaxy with the highest rank. The results were visually inspected and divided into three categories as follows.

1. $\mathrm{CG}-\mathrm{TYPE}=1$ : the CG is visually obvious (for the most part, a dominant early-type galaxy with an extended envelope), and the algorithm has selected the correct galaxy.

2. $\mathrm{CG}-\mathrm{TYPE}=2$ : there is a visual ambiguity in the $\mathrm{CG}$ selection, but we estimate that the algorithm has selected a galaxy that has a $50 \%$ chance of being the CG.

3. CG-TYPE $=3$ : the visual identification is highly ambiguous or there is some other problem that prevents the identification of the CG.

In this study, we only consider CG-TYPE $=1$ and CG-TYPE $=$ 2 systems. In combination with the quality cuts described previously, these cuts leave a total of 118 groups and clusters at $z \leqslant 1$ (95 of which are CG-TYPE $=1$ and 23 are CG-TYPE $=2$ ). The details of the MMCG selection as well as tests regarding centering uncertainties will be presented in a forthcoming paper (M. George et al. 2010, in preparation).

In terms of stacked weak lensing, there are two mis-centering effects to be taken into consideration. The first is that the location of CGs could be poor tracers of the actual centers of their dark matter halos. The second is that the CGs could be misidentified. In a similar fashion to the maxBCG studies (Sheldon et al. 2009; Johnston et al. 2007), we neglect the former. However, our analysis differs from the maxBCG studies with respect to 
the latter. Johnston et al. (2007) assume a CG misidentification fraction of $\sim 30 \%$ and apply a mis-centering kernel in their analysis to account for this effect. In this study, we assume that our X-ray prior combined with thorough visual checks allows us to correctly identify the CG for a majority of our systems and that when a CG is misidentified, the projected radial offset from the dark matter center is not large. Testing this assumption in further detail will be the focus of a subsequent paper (M. George et al. 2010, in preparation). Nonetheless, in Section 7, we have also demonstrated that restricting our analysis to CG-TYPE $=1$ systems does not affect our results, indicating that errors due to mis-centering are probably not a dominant effect for this work.

We also note that our CG selection is based on stellar mass and is insensitive to color; hence we avoid the problem of blue-core BCGs which could represent about $25 \%$ of the BCG population according to Bildfell et al. (2008). The excess blue light in these systems can lead to a typical offset from the red sequence of $0.5-1.0 \mathrm{mag}$ in $\left(g^{\prime}-r^{\prime}\right)$ which could lead to their rejection by red-sequence-type methods.

\section{FUNCTIONAL FORM OF $M-L_{\mathrm{X}}$}

In this section, we present previously published results and overview the various assumptions that are made regarding the functional form of $M-L_{\mathrm{X}}$. We also explain how the scatter between mass and luminosity can cause subtle differences between the study of $M$ as a function of $L_{\mathrm{X}}$ (the " $M-L_{\mathrm{X}}$ relation") and the study of $L_{\mathrm{X}}$ as a function of $M$ (the " $L_{\mathrm{X}}-M$ relation").

Previous measurements of the $L_{\mathrm{X}}-M$ relation based on X-ray data (Reiprich \& Böhringer 2002; Allen et al. 2003; Popesso et al. 2005; Chen et al. 2007; Pratt et al. 2009; Zhang et al. 2008; Vikhlinin et al. 2009) as well as the $M-L_{\mathrm{X}}$ relation derived with lensing (Hoekstra 2007; Bardeau et al. 2007; Rykoff et al. 2008) are by and large consistent with a power law, but with a slope and amplitude that differ from the self-similar prediction of $M \propto L_{\mathrm{X}}^{3 / 4}$. In contrast, the evolution of the $L_{\mathrm{X}}-M$ relation is still under much debate with certain authors finding that the $L_{\mathrm{X}}-M$ relation evolves in a self-similar fashion (Lumb et al. 2004; Arnaud et al. 2005; Kotov \& Vikhlinin 2005; Maughan 2007), while others do not (Ettori et al. 2004).

In addition to the shape and evolution of the mean $L_{X}-M$ relation, astrophysical processes are expected to induce scatter in $L_{X}$ at fixed mass which is important to take into consideration. In the absence of strong observational or theoretical guidance for the form and magnitude of this scatter (although see Reiprich \& Böhringer 2002; Maughan 2007), it is common to adopt a stochastic model where $P\left(L_{\mathrm{X}} \mid M\right)$ is a log-normal probability distribution function (hereafter PDF) with a mean $\log$-luminosity that follows $\left\langle\ln L_{\mathrm{X}}\right\rangle \propto \beta \ln M$ and with a constant $\log$-normal scatter noted $\sigma_{\ln L_{X}}$ (Stanek et al. 2006; Rozo et al. 2009; Rykoff et al. 2008; Vikhlinin et al. 2009). In this particular case, and under the further condition that the halo mass function is a power law, it can be demonstrated that $P\left(M \mid L_{\mathrm{X}}\right)$ is also a log-normal probability function with a dispersion in mass equal to $\sigma_{\ln M}=\sigma_{\ln L_{X}} / \beta$ and a mean log-mass that follows $\langle\ln M\rangle \propto \alpha \ln L_{\mathrm{X}}$, with $\alpha=1 / \beta$ (see the Appendix for further details). As a consequence, the slopes of the $L_{\mathrm{X}}-M$ and the $M-L_{\mathrm{X}}$ relation can be compared quite simply, but the comparison of the normalization will depend on the halo mass function.

The difference in the normalization of $P\left(L_{\mathrm{X}} \mid M\right)$ and $P\left(M \mid L_{\mathrm{X}}\right)$ can be seen as a form of extended Malmquist bias. This is not Malmquist bias is the classical sense because it will occur in any survey, independently of the flux limit. The equations for this bias are derived in the appendix. In general, X-ray astronomers commonly employ $P\left(L_{\mathrm{X}} \mid M\right)$ (Stanek et al. 2006; Vikhlinin et al. 2009), whereas lensing more naturally derives $P\left(M \mid L_{\mathrm{X}}\right)$ (Rozo et al. 2009; Rykoff et al. 2008) so care must be taken when comparing the two.

In reality, the slope of the mass function varies with both mass and redshift and as a consequence, $\alpha=1 / \beta$ no longer holds when slopes are derived over a large range in masses. Corrections for this effect are derived in the appendix.

Stacked weak lensing yields a measurement of the arithmetic mean of the surface mass density contrast, $\langle\Delta \Sigma(r)\rangle$. When the data are binned according to a well chosen proxy and over a narrow redshift range (to avoid smearing the profiles due to evolution in the mass-concentration relation for example), the mass derived by fitting $\langle\Delta \Sigma(r)\rangle$ will be close to the arithmetic mean of the stacked ensemble $\left\langle M_{200}\right\rangle$. For this reason, we select narrow redshift bins that further enable us to assume that $\left\langle M_{200} . E(z)\right\rangle \sim\left\langle M_{200}\right\rangle E(\langle z\rangle)$. Note that if the PDF of the mass at fixed luminosity is log-normal, then $\left\langle M_{200}\right\rangle$ will be different from the peak of the PDF. Indeed, in this case, the peak is traced by the median, not the arithmetic mean. If the scatter of $P(M \mid L)$ is known, then the correspondence between the two is given by $\left\langle M_{200}\right\rangle=\exp \left(\left\langle\ln \left(M_{200}\right)\right\rangle+\sigma_{\ln M}^{2} / 2\right)$. Figure 2 illustrates the various issues outlined above and for which more detailed calculations are presented in the appendix.

Estimates for the scatter ${ }^{29}$ in the $M-L_{\mathrm{X}}$ relation vary from $\sigma_{\ln M} \sim 0.2$ to $\sigma_{\ln M} \sim 0.3$ and can depend exactly on how $L_{\mathrm{X}}$ is measured. The lowest scatter is obtained with cool-core excision techniques (Stanek et al. 2006; Maughan 2007; Pratt et al. 2009). For distant clusters, the cluster core region can become smaller than the observed PSF and so cool-core excision becomes infeasible. However, there have been suggestions that the coolcore fraction is low at $z>0.5$ (Vikhlinin et al. 2007), perhaps making excision unnecessary at higher redshifts. In addition, Maughan (2007) have suggested that $\sigma_{\ln M}$ is reasonably small even for survey quality data. In any case, although the scatter in mass at fixed luminosity is still poorly constrained (especially at high redshift), by most estimates it is smaller than the scatter in mass at fixed richness, even for the best richness estimators (e.g., Rozo et al. 2009, find $\sigma_{\ln M \mid \lambda}=0.45$ ).

Given the various considerations discussed above, we adopt a power-law form for the mean relation between mass and luminosity with a redshift evolution that follows self-similarity:

$$
\frac{\left\langle M_{200} E(z)\right\rangle}{M_{0}}=A\left(\frac{\left\langle L_{X} E(z)^{-1}\right\rangle}{L_{X, 0}}\right)^{\alpha},
$$

where $M_{0}=10^{13.7} h_{72}^{-1} M_{\odot}$ and $L_{X, 0}=10^{42.7} h_{72}^{-2} \mathrm{erg} \mathrm{s}^{-1}$. Deviations from self-similar evolution are tested for in Section 6.4.

To begin with, we derive the relationship between the mean mass $\left\langle M_{200}\right\rangle E(\langle z\rangle)$ and the mean luminosity $\left\langle L_{X} E(z)^{-1}\right\rangle$ using only the COSMOS data. This relation is referred to as $\mathcal{R} 1_{M-L_{X}}$. Next, we combine the COSMOS results with previously published cluster data to improve measurements of the slope $\alpha$. This combined relation is referred to as $\mathcal{R} 2_{M-L_{X}}$.

\section{RESULTS}

In this section, we present our lensing measurements as well as the $M-L_{X}$ relations $\mathcal{R} 1_{M-L_{X}}$ and $\mathcal{R} 2_{M-L_{X}}$. We also test for

\footnotetext{
${ }^{29}$ Scatter is quoted as the standard deviation of the natural logarithm of the mass at fixed $L_{X}$.
} 

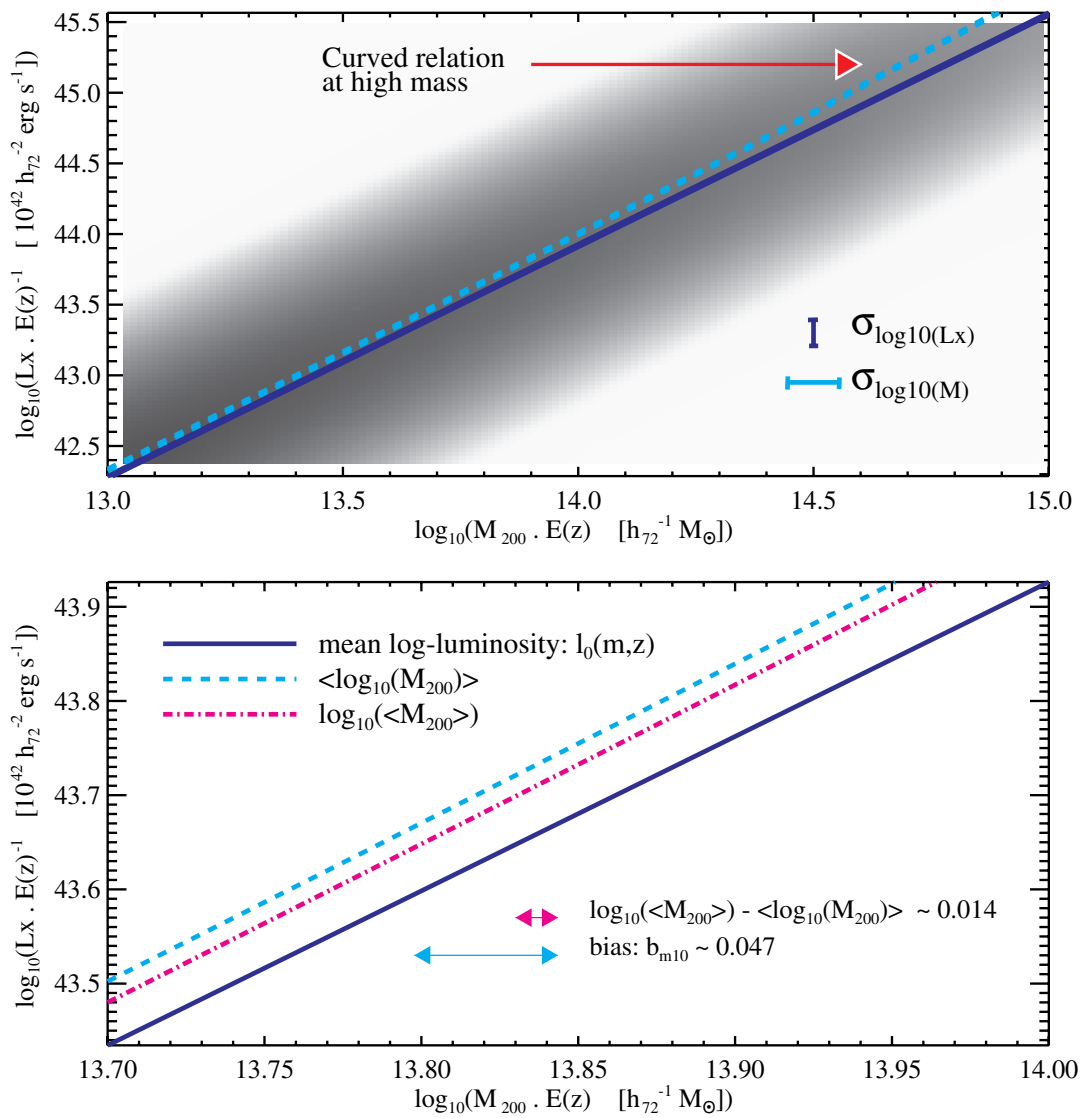

Figure 2. Illustration of the effects of biases in the $L_{\mathrm{X}}-M$ and the $M-L_{\mathrm{X}}$ relations at $z=0.2$. The conditional probability distribution of the luminosity given the mass, $P\left(L_{X} \mid M, z\right)$, is assumed to be log-normal with a mean log-luminosity that follows a power-law scaling relation (blue solid line) with a slope of $1.63=1 / 0.61$ and with a scatter of $\sigma_{\ln l}=0.4$. A simulated ensemble of groups and clusters of galaxies is created for which the number densities per mass follow the Tinker et al (2008) halo mass function and for which X-ray luminosities are attributed according to $P\left(L_{\mathrm{X}} \mid M, z\right)$. The gray shading in the upper panel is proportional to the log of the number density of groups and clusters in the luminosity-mass plane (arbitrary normalization). The mean log mass follows the cyan dashed line and the log of the mean mass $\left(\log _{10}\left\langle M_{200}\right\rangle\right.$, measured by lensing $)$ is shown by the dash-dot magenta line. There is a mass-dependent bias between the cyan and the dark blue line equal to $b_{m}=\sigma_{m}^{2}(\gamma-1)$, where $\gamma$ is the slope of the mass function (see the Appendix). As a results of this bias, there is a slight curvature in both the cyan and the magenta lines at high halo masses.

(A color version of this figure is available in the online journal.)

additional redshift evolution in the $M-L_{\mathrm{X}}$ relation beyond that predicted by self-similarity.

\subsection{Stacked Estimates of $\Delta \Sigma$ and $M_{200}$}

The data are divided into nine bins labeled $A_{0}$ through $A_{8}$ (see Figure 3 and Table 1). The bins are selected to encompass a narrow range in redshift and $L_{X} E(z)^{-1}$ so as to avoid smearing out the signal due to evolution in the mass-concentration relation. For each bin, the stacked weak lensing signal is calculated according to the method outlined in Section 3.

We calculate the weak lensing signal from $40 \mathrm{kpc}$ to $4 \mathrm{Mpc}$ in logarithmically spaced radial bins of 0.26 dex. A weak lensing signal is detected all the way to $4 \mathrm{Mpc}$, allowing us to probe the full extent of the one-halo term. The results are fit with the parametric model given by Equation (12).

We use a Markov chain Monte Carlo (MCMC) method to fit the $\Delta \Sigma$ profiles. The MCMC routine uses the MetropolisHastings algorithm with a Gaussian transfer function. The total number of steps is 30,000 , and a burn-in period of 500 is discarded. Bins with less than 15 background sources in total are excluded from the fit.

The results from the stacked analysis and the fits to the profiles are shown in Figure 4. At the smallest radii that we probe, the stellar mass of the CG plays a minor role in the lensing signal but we have added it for consistency. The scales that we probe are dominated by the signal due to the dark matter halo associated with the groups. Our estimates for $\left\langle M_{200}\right\rangle$ are given in Table 1.

\subsection{Measurement of $\mathcal{R} 1_{M-L_{X}}$}

Fitting only the low redshift COSMOS data (from A0 to A6, $0.2<z<0.5$ ), we obtain the best-fit parameters $\log _{10}(A)=0.068 \pm 0.063$ and $\alpha=0.66 \pm 0.14$ (see Figure 5). The cited errors are statistical only. The effects of systematic errors are explored in Section 7 and are estimated to be lower than the statistical uncertainty. The cool-core correction factor that we apply does not strongly affect these results. Without the cool-core correction, we obtain $\log _{10}(A)=0.09 \pm 0.062$ and $\alpha=0.64 \pm 0.14$.

Figure 6 compares the COSMOS $\mathcal{R} 1_{M-L_{X}}$ relation to four other lensing-based measurements: the Sloan Digital Sky Survey (SDSS) results from Rykoff et al. (2008, hereafter R08), four groups from Bergé et al. (2008, hereafter BE08), cluster data from Hoekstra (2007, hereafter H07) with masses updated in Madhavi et al. (2008), and cluster data from Bardeau et al. (2007, hereafter BA07). All data points have been normalized to $H_{0}=72 h_{72} \mathrm{~km} \mathrm{~s}^{-1} \mathrm{Mpc}^{-1}$ and scaled by $E(z)$. The cluster data points from BA07 and the $\mathrm{H} 07$ have been selected on the basis that their lensing analysis extends to the virial radius, allowing them to derive mass estimates that are comparable to ours. Each of these four independent studies probes a distinct redshift and 


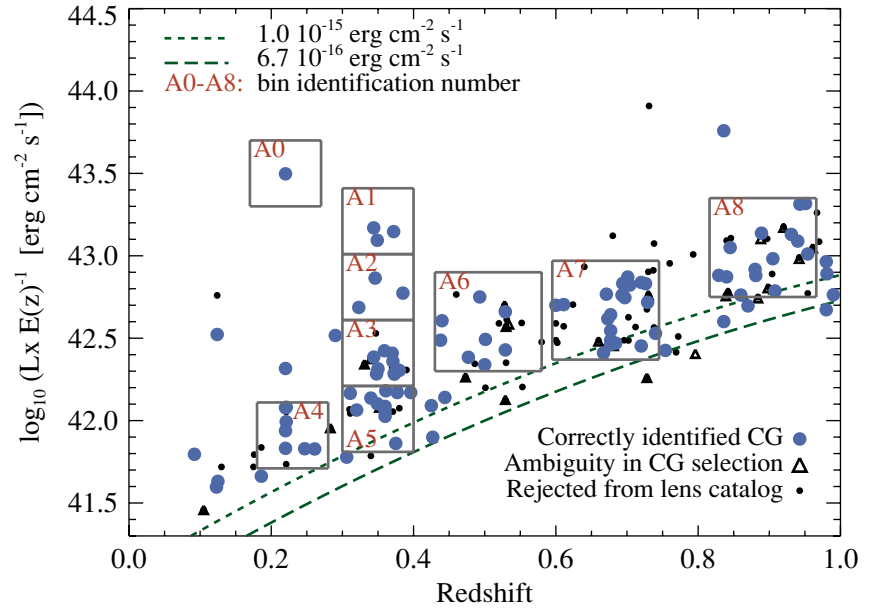

Figure 3. COSMOS groups within the ACS field as a function of redshift and $L_{\mathrm{X}} E(z)^{-1}$, binned into nine sub-samples labeled $A_{0}$ through $A_{8}$. Each bin is designed to encompass a narrow range in both redshift and $L_{X} E(z)^{-1}$. The $4 \sigma$ sensitivity limits are shown by the green lines: $96 \%$ of the ACS field is covered to a sensitivity limit of $1.0 \times 10^{-15} \mathrm{erg} \mathrm{cm}^{-2} \mathrm{~s}^{-1}$ where the sensitivity limits have been derived using a wavelet sensitivity map on group scales (green dotted line), and $52 \%$ of the ACS field is covered to a deeper limit of $6.7 \times 10^{-16}$ erg $\mathrm{cm}^{-2} \mathrm{~s}^{-1}$ (green dashed line). Small black circles represent systems that were rejected from the lens catalog. Blue-filled circles indicate systems for which the central galaxy (CG) identification is certain, whereas black triangles show systems for which the identification is more ambiguous.

(A color version of this figure is available in the online journal.)

mass scale. Nevertheless, the ensemble of data points displays a remarkable trend that spans over three orders of magnitude in $L_{X} E(z)^{-1}$ and two orders of magnitude in $M_{200} E(z)$. The varying degrees of scatter seen between the different data sets are due to the fact that some results are direct detections of individual clusters (e.g., H07, BA07, and BE08), while other data points have been stacked (e.g., R08). The COSMOS data points are scattered about the mean relation because each bin contains a relatively small number of groups (tens of groups as opposed to hundreds in R08). We will now briefly describe each of these data sets. Further discussion of the agreement between various results is presented in Section 8.

The R08 data points (light blue, plus signs) are taken from Table 1 of their paper. $L_{\mathrm{X}}$ and $M_{200}$ have been normalized to our adopted value of $H_{0}$ and scaled with the $E(z)$ factor at the quoted redshift of $z=0.25$. Mandelbaum et al. (2008) have shown that the masses published by Johnston et al. (2007) which have been used in the R08 analysis must be boosted by a factor of $1.18^{1.4}=1.25$ when the SDSS source distribution is calibrated against zCOSMOS spectroscopic redshifts. We boost the R08 data points by a factor of 1.24 (a revised version of the published correction; R. Mandelbaum 2009, private communication), and the results are shown by the small black triangles in Figure 6 . The upper error bars of the R08 data points are increased in order to reflect this correction. Note that because both $M_{200}$ and $L_{X}$ have been derived via stacking methods, the results of R08 will depend on the covariance between $L_{X}$ and richness (noted $N_{200}$, see R08) at fixed mass: the slope of their relation will change depending on the correlation between these two parameters. This is not an issue for COSMOS where the stacking is performed directly on $L_{\mathrm{X}}$ instead of using an intermediate variable such as $N_{200}$.

The masses for the BE08 data points (dark green, asterisk signs) are taken from their paper, and $L_{X}$ has been provided by F. Pacaud (2009, private communication.).
The masses for the BA07 data points (sienna, cross signs) are taken from their paper. X-ray luminosities are derived from the XMM LOCUSS survey using the same X-ray data as in Zhang et al. (2008) but by integrating the flux out to the truncation radius of $2.5 r_{500}^{Y_{X}, w l}$. Note that $r_{500}^{Y_{X}, w l}$ is given in Section 6.2.3 of Zhang et al. (2008). The imaging data for the BA07 analysis are based on ground-based wide-field imaging obtained with the CFH12k camera on the Canada-France-Hawaii Telescope. One cluster in the BA07 paper did not have an XMM LOCUSS luminosity and has been discarded (namely A2219).

The $\mathrm{H} 07$ data points (dark blue diamonds) have been taken from Column 8 of Table 1 in Madhavi et al. (2008). Luminosities are derived from the LOCUSS survey using the methodology described in the previous paragraph. The overlap between the surveys is the following set of clusters: A68, A209, A267, A383, A963, A1689, A1763, A2218, and A2390. Note that BA07 and H07 have studied a common set of clusters using the same imaging data (CFH12k camera). Differences is cluster mass estimates between BA07 and $\mathrm{H} 07$ are probably due to dissimilar data analysis techniques (such as assumptions regarding the mass-concentration relation for example).

\subsection{Measurement of $\mathcal{R} 2_{M-L_{X}}$}

In this section, we perform a joint fit between the COSMOS data and cluster data from $\mathrm{H} 07$ and BA07. The high redshift COSMOS data points (bins $A_{7}$ and $A_{8}$ ) are excluded from this fit so that all data points are at a comparable redshift $(z \sim 0.3)$. The joint fit COSMOS $/ \mathrm{H} 07$ yields $\log _{10}(A)=0.03 \pm 0.06$ and $\alpha=0.64 \pm 0.03$. The joint fit COSMOS/B07 also yields $\log _{10}(A)=0.03 \pm 0.06$ and $\alpha=0.64 \pm 0.03$. These results raise several points of interest. First, the fit to the COSMOS data alone gives a very similar relation to the fit when the cluster data are added. This suggests that the $M-L_{\mathrm{X}}$ relation is invariant from group to cluster scales with no detected break at group scales. Second, the combination of group and cluster data creates a large dynamic mass range which allows for a 5\% (statistical error) determination of the slope of the $M-L_{\mathrm{X}}$ relation. Finally, despite the systematic differences in the mass estimates of $\mathrm{H} 07$ and BA07, the combined relations with COSMOS are identical. The addition of group data has significantly reduced the impact of cluster mass uncertainties on the measured $M-L_{\mathrm{X}}$ relation.

\subsection{Redshift Evolution in the $M-L_{X}$ Relation}

In this section, we test for redshift evolution in the $M-L_{X}$ relation by adding an additional redshift-dependent term to our previously assumed $M-L_{\mathrm{X}}$ relation as follows:

$$
\begin{gathered}
\frac{\left\langle M_{200} E(z)\right\rangle}{M_{0}}=A\left(\frac{\left\langle L_{X} E(z)^{-1}\right\rangle}{L_{X, 0}}\right)^{\alpha} E(z)^{\gamma} \\
\frac{\left\langle M_{200} E(z)\right\rangle}{M_{0}}=A\left(\frac{\left\langle L_{X} E(z)^{-1}\right\rangle}{L_{X, 0}}\right)^{\alpha}(1+z)^{\delta} .
\end{gathered}
$$

Fitting the COSMOS data alone with Equation (14) yields $\log _{10}(A)=0.05 \pm 0.1, \alpha=0.70 \pm 0.13$, and $\gamma=-0.07 \pm 0.9$. Equation (15) yields $\log _{10}(A)=0.07 \pm 0.14, \alpha=0.70 \pm 0.13$, and $\delta=-0.14 \pm 0.8$. In both cases, the COSMOS data are consistent with self-similar redshift evolution to the level that can be probed with these data. Additional cluster and group data with weak-lensing masses at $0.6<z<1.0$ would be required in order to constrain the redshift evolution in the $M-L_{\mathrm{X}}$ relation to higher precision. 


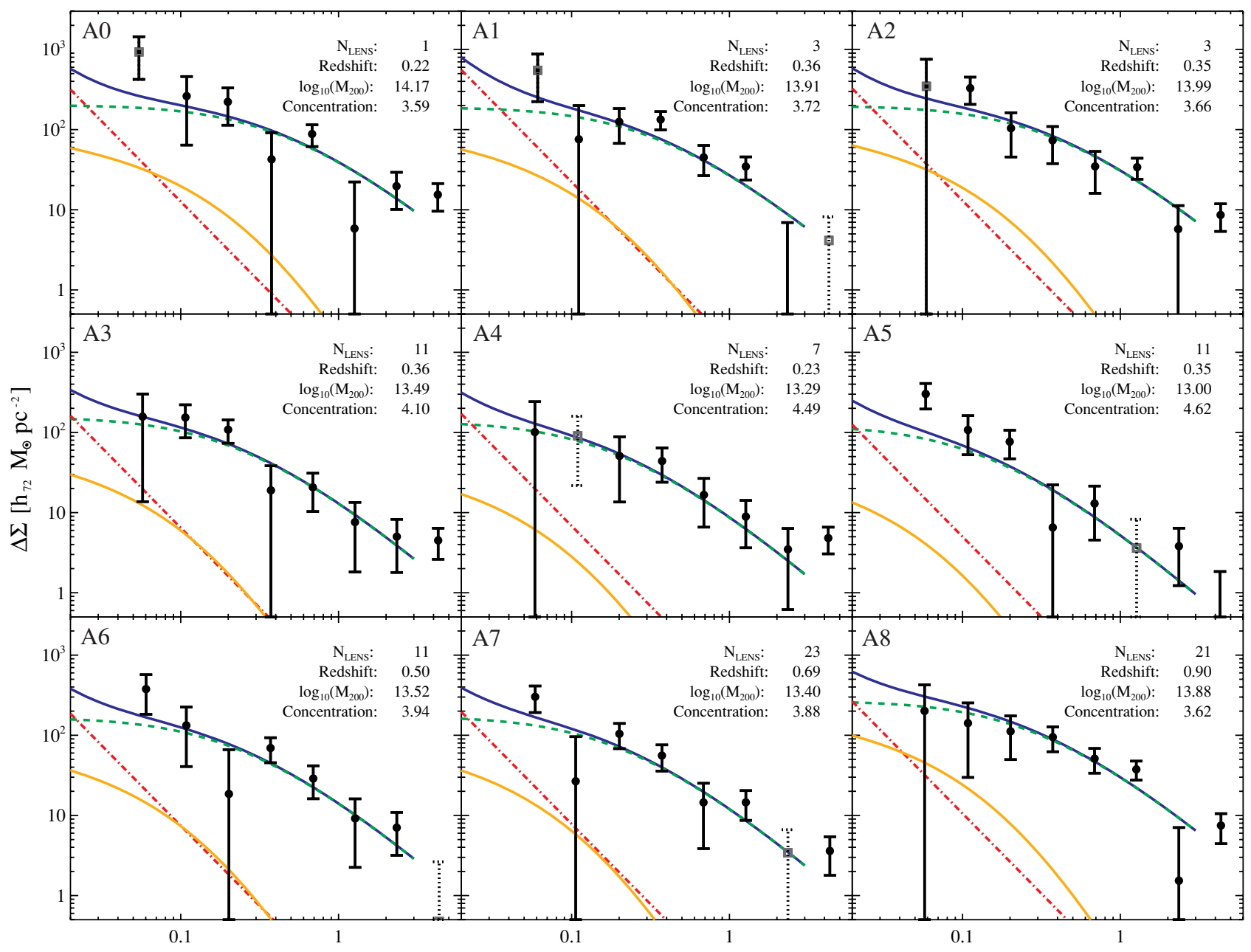

Physical transverse distance, $\mathrm{R}\left[\mathrm{Mpc} \mathrm{h} \mathrm{h}_{72}^{-1}\right]$

Figure 4. Stacked weak lensing profiles of COSMOS groups for nine bins that each span a narrow range in redshift and $L_{X} E(z)^{-1}$. From left to right and top to bottom we show the bins $A_{0}$ through $A_{8}$. A weak lensing signal is detected all the way to $4 \mathrm{Mpc}$, allowing us to probe the lens density profiles well beyond the virial radius. The solid blue curve shows our fit to the data which is the sum of a baryonic term (red dash-dot), an NFW profile (green dash-dash), and a second-order weak shear correction term (orange dash-dot-dot, see Section 3.2). Gray points are negative data points and bins with less than 15 source galaxies.

(A color version of this figure is available in the online journal.)

\section{ASSESSMENT OF SYSTEMATICS ERRORS}

There are three potential causes of systematic errors in this work: errors in the photometric redshifts, mis-centering, and uncertainty in the mass-concentration relation. As demonstrated below, we have found that the systematic errors associated with each of these effects are below the statistical error, at least to the extent that this can be tested for with the current data.

\subsection{The Impact of Photometric Redshift Errors}

The effects of redshift errors on group-galaxy lensing signals can be broadly categorized as follows: (1) uncertainties in the redshifts of the lenses will smear out the signal and affect the derivation of $\Sigma_{\text {crit }}$; (2) errors in the mean source redshift distribution will introduce a bias in the normalization of the overall signal; (3) improper lens-source separation will lead to a dilution of the signal and will subject the signal to the effects of intrinsic alignments; and (4) catastrophic errors can also dilute the signal. In this work, we neglect (1) on the basis that the redshifts of the groups are well determined $(81 \%$ have two or more spectroscopically confirmed members).

To reduce the effects of (3) and (4), we use the full redshift PDF which is derived by the photoz code LePhare for each

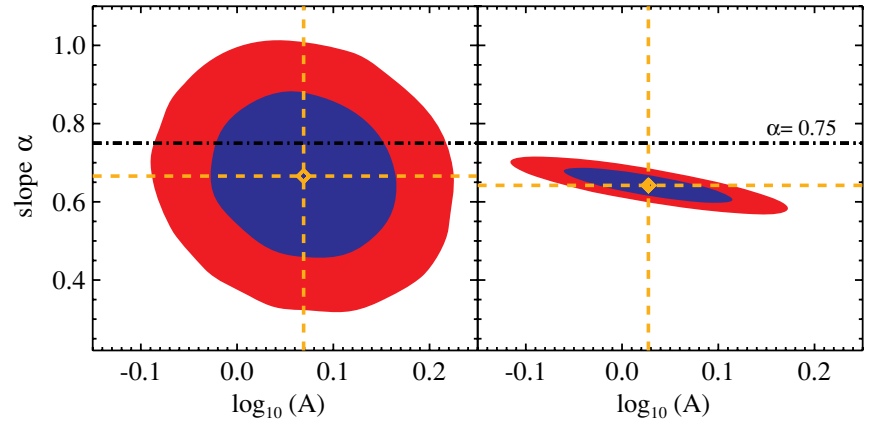

Figure 5. Left panel: marginal posterior distribution for the parameters of $\mathcal{R} 1_{M-L_{X}}$ (COSMOS data only). The blue-shaded region denotes the $68 \%(1 \sigma)$ confidence region and the red-shaded region denotes the $95 \%(2 \sigma)$ confidence region. The dash-dot line indicates the self-similar prediction for the slope of the $M-L_{\mathrm{X}}$ relation. Right panel: marginal posterior distribution for the combination of the COSMOS group data and cluster data from Hoekstra (2007). The large dynamic mass range created by the combination of group and cluster data enables a more accurate determination of the slope of the $M-L_{\mathrm{X}}$ relation.

(A color version of this figure is available in the online journal.)

source galaxy (Ilbert et al. 2009). The main peak in the PDF, ZP_MAX, represents the most probable redshift. When present, 


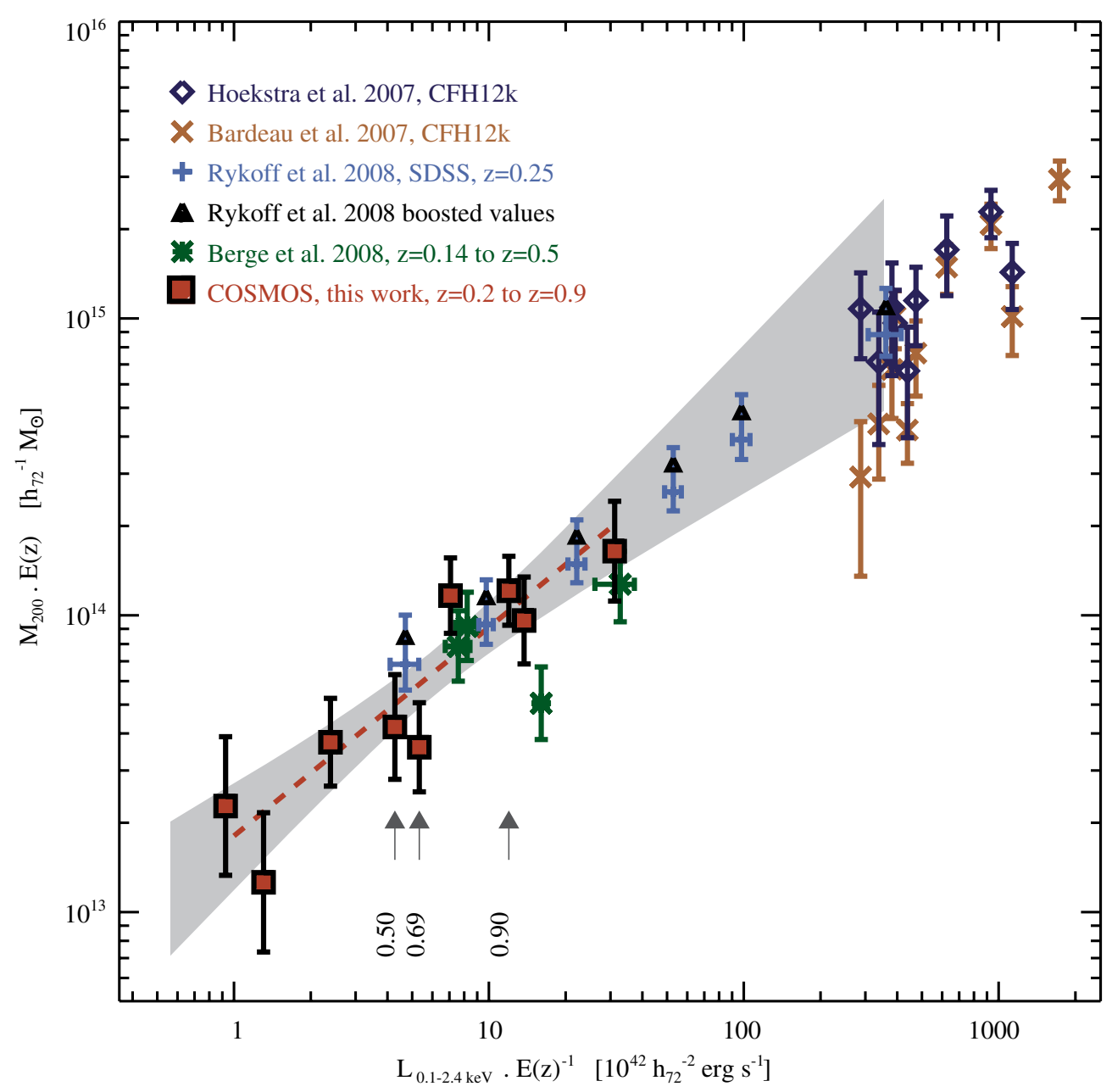

Figure 6. COSMOS $M-L_{\mathrm{X}}$ relation. Dark blue diamonds show individually detected clusters from H07 with updated masses from Madhavi et al. (2008). Sienna cross symbols show data points from BA07. Light blue plus symbols represent the R08 results from a stacked analysis in the SDSS and black diamonds take into account a recent correction to these masses due to a new calibration of the source distribution. The upper error bars have been adjusted to account for the redshift uncertainty. Green asterisks show four data points at intermediate masses from BE08. Finally, the red squares depict our COSMOS results which extend previous results to lower masses and to higher redshifts. Three arrows highlight the highest redshift COSMOS data points (bins $A_{6}, A_{7}$, and $A_{8}$ ). The gray-shaded region shows the upper and lower envelope of the ensemble of lines with a slope and intercept that lie within the 68 percent confidence region of $\mathcal{R} 1_{M-L_{X}}$.

(A color version of this figure is available in the online journal.)

a secondary peak in the PDF is noted ZP_SEC. The galaxy population with double peaked PDFs is expected to contain a large fraction of catastrophic errors (roughly $40 \%-50 \%$, Ilbert et al. 2006, 2009). In this work, we eliminate sources with a double peaked PDF in order to minimize the effects of signal dilution caused by catastrophic errors. Making this photoz cut leads to a decreased background number density of 34 galaxies $\operatorname{arcmin}^{-2}$.

The redshift PDF information is also used to improve the lens-source separation by using the lower $68 \%$ confidence bound on the source redshift. Sources are selected such that $z_{S}-z_{L}>\sigma_{68 \%}\left(z_{S}\right)$. It is also important to note that groupgalaxy lensing signals are most sensitive to redshift errors when $z_{S}$ is only slightly larger then $z_{L}$ (see Figure 8). For this reason, in addition to the previous cut, we also implement a fixed cut such that $z_{S}-z_{L}>\delta z$, where $\delta z$ is defined below in the following two schemes.

1. $\mathcal{S} 1$ : only sources with a single-peaked PDF are used, $z_{S}-z_{L}>\sigma_{68 \%}\left(z_{S}\right)$, and $z_{S}-z_{L}>0.1$ (the default scheme used throughout this paper).

2. $\mathcal{S} 2$ : only sources with a single-peaked PDF are used, $z_{S}-z_{L}>\sigma_{68 \%}\left(z_{S}\right)$, and $z_{S}-z_{L}>0.2$.
We test each of these two schemes, and the results are shown in Figure 7. Choosing a value of $\delta z=0.1$ or $\delta z=0.2$ has a negligible impact on $M_{200}$.

In order to quantify the errors associated with (2) and (4), we make use of the ensemble of spectroscopic redshifts that are currently available from the zCOSMOS program. As illustrated in Figure 8, source galaxies with $z_{\text {phot }}>z_{\text {lens }}$ and $z_{\text {spec }}<z_{\text {lens }}$ dilute the signal, whereas galaxies with $z_{\text {phot }}>z_{\text {lens }}$ and $z_{\text {spec }}>z_{\text {lens }}$ but $z_{\text {phot }} \neq z_{\text {spec }}$ will introduce a bias in $\Delta \Sigma$ because $\Sigma_{\text {crit }}$ will be mis-estimated when transforming $\gamma$ into $\Delta \Sigma$. The correction factor for biases in the photometric redshifts is noted $f_{\text {bias }}$, while $f_{\text {boost }}$ represents a number greater than 1 that boosts the measured signal to compensate for signal dilution. The true signal is related to the measured one according to

$$
\Delta \Sigma_{\text {true }}=\Delta \Sigma_{\text {meas }} \times f_{\text {bias }} \times f_{\text {boost }} \text {. }
$$

Using the ensemble of zCOSMOS spectra that are currently available, we have estimated $f_{\text {bias }}$ and $f_{\text {boost }}$ for each of our nine bins. The maximum bias that we find is a $7 \%$ upward correction on $\Delta \Sigma$ for the last redshift bin which corresponds to a revised mass of $8.5 \times 10^{13} h_{72}^{-1} M_{\odot}$. This is a small correction compared to our measured value of $7.6_{-1.8}^{+2.3} \times 10^{13} h_{72}^{-1} M_{\odot}$. In conclusion, 


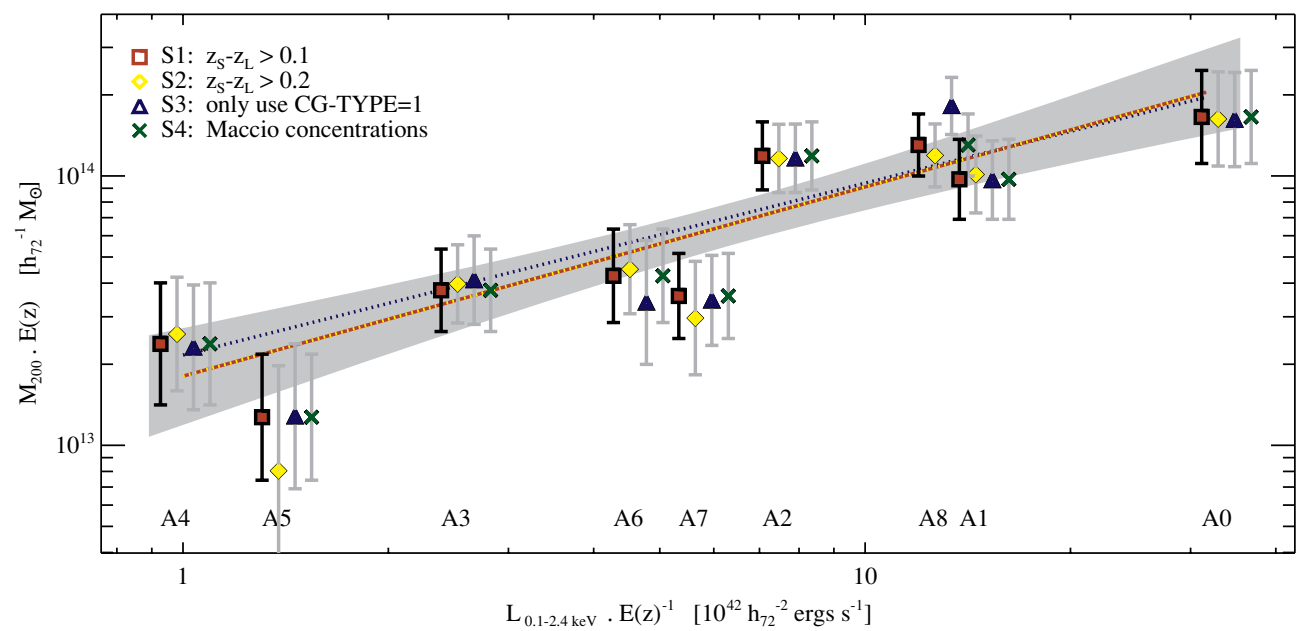

Figure 7. Tests for systematic errors. We implement four tests that are designed to check for errors in the foreground-background separation scheme to probe the effects of mis-centering and to quantify the impacts of variations in the mass-concentration relation. The lensing signal is recomputed for each test, and the results are compared to the masses used to calculate $\mathcal{R} 1_{L_{X}-M}$. The data points have been offset on the $X$-axis by a constant value for visualization purposes. The gray-shaded region shows the upper and lower envelopes of the ensemble of lines with a slope and intercept that lie within the 68 percent confidence region of $\mathcal{R} 1_{L_{X}}-M$. All the effects that are mentioned above are found to have a negligible impact on the results.

(A color version of this figure is available in the online journal.)

to the extent that we can estimate $f_{\text {bias }}$ and $f_{\text {boost }}$ using the current zCOSMOS data, we find that the errors due to imperfect photometric redshifts are below the statistical uncertainties. It is important to note, however, that the zCOSMOS spectroscopic sample may not be fully representative of the background source population because of incompleteness. Thus, it is possible that our estimates of $f_{\text {bias }}$ and $f_{\text {boost }}$ are erroneous. For this reason, we do not apply these correction factors to the data and have simply listed the values of $f_{\text {bias }}$ and $f_{\text {boost }}$ in Table 1 as an indication of the probable systematic uncertainty due to photometric redshift errors.

\subsection{The Effects of Mis-centering}

To test for mis-centering effects, we recalculate the lensing signals using only systems with CG-TYPE $=1$ instead of using both $\mathrm{CG}$-TYPE $=1$ and CG-TYPE $=2$. The results are shown in Figure 7, and we find that restricting our analysis to the subsample of groups that have visually obvious CGs has no impact on our estimates of $M_{200}$.

\subsection{The Mass-Concentration Relation}

Testing for the effects of theoretical uncertainties in the $M_{200}-$ $C_{200}$ relation is beyond the scope of this paper; however, one test we can perform is whether or not different $M_{200}-C_{200}$ relations affect our mass estimates. For this purpose, we compare two recently derived $M_{200}-C_{200}$ relations, one from Macciò et al. (2007) and the second from Zhao et al. (2008). We compute our lensing signals with each of these relations and show that the results are largely unaffected by this test. Note that the agreement in the $M_{200}-C_{200}$ relation from various authors is fairly good in the mass and redshift regime of our group sample (the typical fractional difference is 10\%-20\%). However, at higher masses the disagreement is larger and hence the assumed concentrations in $\mathrm{H} 07$ and BA07 could represent a systematic error in the joint fit between COSMOS and the cluster data.

\subsection{Conclusions Regarding Systematic Uncertainties}

As demonstrated in Figure 7, all the effects that we have tested for are largely negligible compared to the statistical error.
However, one aspect that we have not explored in this work is the fact that each of our stacks contains a relatively small number of groups. Indeed, the assumption of spherical symmetry will begin to break down for stacks that only contain a small number of objects and the weak lensing signal may be contaminated from projection effects that have not averaged away. We have tried to limit this effect by discarding all systems with visible projections along the line of sight. In total, the various quality cuts described in Section 4 are such that about $30 \%$ of the initial sample is rejected from the lens catalog. Most of these cuts were linked to the quality of the X-ray data and to projection effects. Additional XMM-Newton and Chandra data would increase the size of our lens sample and would help reduce the statistical error on the mass measurement.

\section{SUMMARY AND DISCUSSION}

In this work, we have used a sample of 206 X-ray-detected galaxy groups to investigate the scaling relation between total mass and X-ray luminosity where $M_{200}$ is derived via weak gravitational lensing. In the following paragraphs, we present a summary and discussion of our main results.

The combination of group and cluster data. The COSMOS group catalog spans an approximate mass and redshift range $M_{200} \sim 10^{13} h_{72}^{-1} M_{\odot}-M_{200} \sim 10^{14} h_{72}^{-1} M_{\odot}$ with $0.2<z<$ 0.9 , a new parameter space in terms of weak lensing-based mass measurements of X-ray-detected groups and clusters of galaxies. When appropriately scaled for self-similar redshift evolution, the COSMOS data, alongside previously published results display a remarkable power-law relation that spans over three orders of magnitude in $L_{X} E(z)^{-1}$ and two orders of magnitude in $M_{200} E(z)$. The COSMOS data alone are well fit by a power law, $M_{200} \propto\left(L_{X}\right)^{\alpha}$, with a slope of $\alpha=0.66 \pm 0.14$. By combining with previously published cluster data, we derive a tighter constraint on the slope, $\alpha=0.64 \pm 0.03$. This is inconsistent at the $3.7 \sigma$ level with the self-similar prediction of $\alpha=0.75$. Note that the combination of group and cluster data greatly helps constrain the slope of the $M-L_{\mathrm{X}}$ relation but the determination of the normalization is at present limited by the accuracy of weak lensing measurements. Improvements in weak lensing methods and larger group and cluster samples will be 

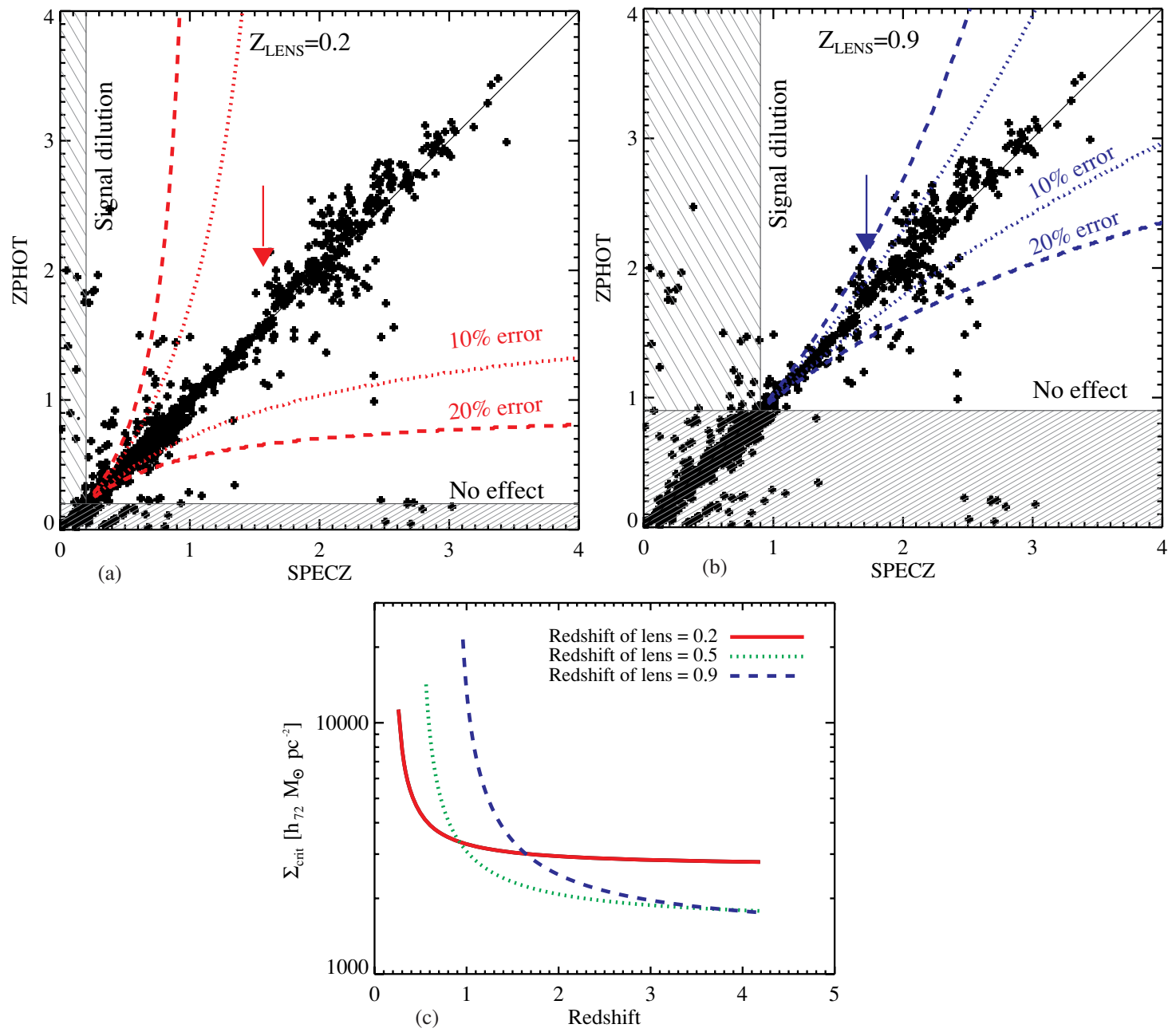

Figure 8. Effects of photometric redshifts errors on group-galaxy lensing signals. Panels (a) and (b) illustrate the quality of the photometric redshifts used in this paper by comparing them to a combined sample of 12367 spectroscopic redshifts from the zCOSMOS "bright" and "faint" programs. There are three ways in which photometric redshift errors can impact group-galaxy lensing signals. First, any type of photometric error such that $z_{\text {phot }}<z_{\text {lens }}$ will have no effect on the signal because such objects are not included in the background selection (bottom hashed region). Second, photometric errors such that $z_{\text {phot }}>z_{\text {lens }}$ and $z_{\text {spec }}<z_{\text {lens }}$ will lead to a signal dilution (left hashed region). Finally, photometric errors such that $z_{\text {phot }}>z_{\text {lens }}$ and $z_{\text {spec }}>z_{\text {lens }}$ but $z_{\text {phot }} \neq z_{\text {spec }}$ will lead to a bias in $\Delta \Sigma$ because $\Sigma_{\text {crit }}$ will be mis-estimated when transforming $\gamma$ into $\Delta \Sigma$. The dotted and dashed lines in panels (a) and (b) indicate where photoz errors lead to a $10 \%$ and a $20 \%$ errors on $\Delta \Sigma$. As can be seen, group-galaxy lensing signals are increasingly insensitive to photometric redshift errors at higher source redshifts. This can be understood by looking at the variation of $\Sigma_{\text {crit }}$ as a function of source redshift as shown in panel (c). Indeed, $\Sigma_{\text {crit }}$ varies strongly near $z_{\text {lens }}$ but flattens considerably at $z_{\text {source }}>2 z_{\text {lens }}$. The arrows in panels (a) and (b) show where the mean COSMOS source redshift lies for lenses at $z_{\text {lens }}=0.2$ and $z_{\text {lens }}=0.9$. The bias in $\Delta \Sigma$ for group-galaxy lensing in COSMOS is estimated to be less than $7 \%$ for lenses below $z=1$.

(A color version of this figure is available in the online journal.)

necessary in order to improve constraints on the normalization of the $M-L_{\mathrm{X}}$ relation.

Comparison with previous lensing results. Our analysis compares best with the local SDSS results from R08 who find $L_{\mathrm{X}} \propto\left(M_{200}\right)^{\beta}$ with $\beta=1.65 \pm 0.13$. The inverse of their slope, $1 / \beta=0.61 \pm 0.048$, is in excellent agreement with both $\mathcal{R} 1_{M-L_{X}}$ and $\mathcal{R} 2_{M-L_{X}}$. As described in their paper, because the R08 results are binned by richness, the slope of their relation is sensitive to the correlation coefficient between richness and $L_{\mathrm{X}}$ at fixed mass, $r_{N, L \mid M}$ (see Section 4 in R08). A value of $r_{N, L \mid M}= \pm 0.7$ would change their slope to $0.68 \pm 0.061$ and $0.54 \pm 0.038$, respectively. These slopes would still be in relatively good agreement with the COSMOS results but much larger values than $r_{N, L \mid M}= \pm 0.7$ can be ruled out. Small values for $r_{N, L \mid M}$ are also favored by the analysis of Rozo et al. (2009), who find a value of $r_{N, L \mid M} \sim 0.05$ (E. Rozo 2010, private communication).

BA07 have also published a $M-L_{\mathrm{X}}$ relation but find a slope of $1.20 \pm 0.16$ that is inconsistent with the COSMOS value at the 3.4 $\sigma$ level. We suspect that this disagreement stems from an underestimate of the BA07 masses, in particular at the low-mass end of the cluster sample. Indeed, H07 have analyzed almost exactly the same set of clusters but find a slope and normalization that is in better agreement with both this work and R08 (see Figure 6 and also Figure 9). Nevertheless, despite systematic differences between the mass estimates of $\mathrm{H} 07$ and BA07, the combined relations using either data set with COSMOS are almost identical. Manifestly, the addition of group data has significantly reduced the impact of cluster mass uncertainties on the $M-L_{\mathrm{X}}$ relation.

Comparison with previous $X$-ray results. The comparison of the normalization of the $M-L_{\mathrm{X}}$ relation derived with lensing on the one hand and with X-rays on the other hand is of great interest because it has the potential to reveal systematic biases in X-ray-based cluster mass estimates (e.g., due to non-thermal processes such as turbulence and cosmic rays). However, the comparison of the normalization between the $M-L_{\mathrm{X}}$ relation and the $L_{\mathrm{X}}-M$ relation is complex because 


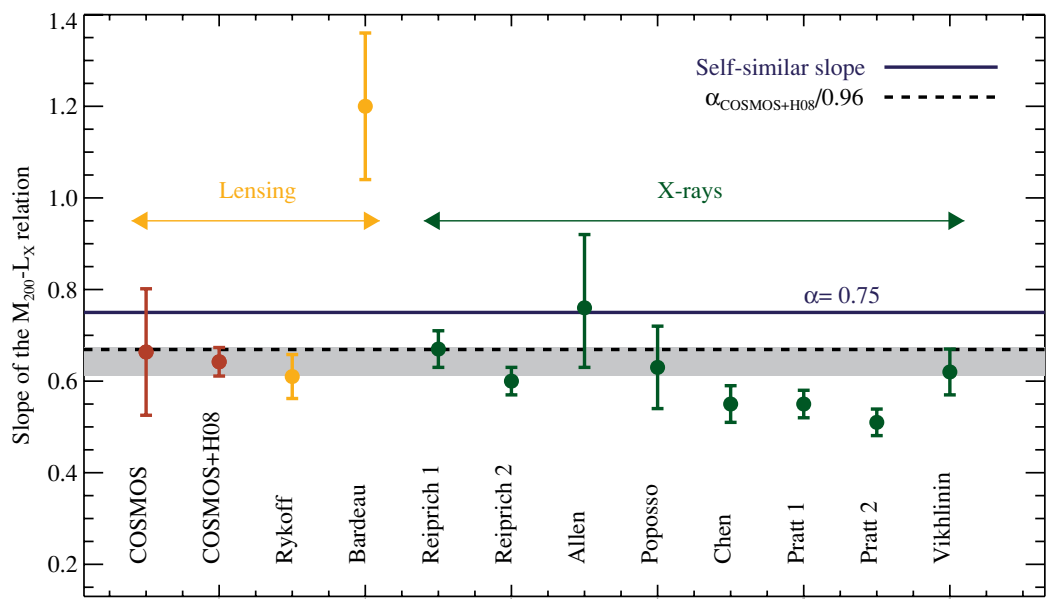

Figure 9. Comparison of the slope of the $M-L_{\mathrm{X}}$ relation obtained by various authors. From left to right we show $\mathcal{R} 1_{M-L_{X}}$ and $\mathcal{R} 2_{M-L_{X}}$ (this work) followed by the lensing based results of Rykoff et al. (2008) and Bardeau et al. (2007) and then by X-ray-based results (Reiprich \& Böhringer 2002; Allen et al. 2003; Popesso et al. 2005; Chen et al. 2007; Pratt et al. 2008; Vikhlinin et al. 2009). The solid blue shows the self-similar prediction for the slope which is $\alpha=0.75$. The gray-shaded region indicates the one sigma errors for $\mathcal{R} 2_{M-L_{X}}$. With the exception of the BA07 results, the lensing and the X-ray results are in good agreement with an average slope of $\alpha \sim 0.64$. Note that because of scatter in the $L_{X}-M$ relation and a halo mass function with a varying slope, the lensing and the X-ray results are not directly comparable. The dashed black line indicates the value of the COSMOS+H08 data point corrected for the difference between $1 / \beta$ and $\alpha_{\text {lensing }}$ assuming a scatter of $\sigma_{\ln M}=0.25$ (see derivations in the appendix).

(A color version of this figure is available in the online journal.)

it depends on $\sigma_{\ln M}$ and the slope of the halo mass function (see the Appendix) and we leave this aspect for future work. Therefore, we mainly focus on a comparison of the slopes. For this purpose, we have compiled a list of the slopes of the $L_{\mathrm{X}}-M$ relation as determined by various $\mathrm{X}$-ray studies. When authors have stated their results in terms of the $L_{X}-M$ relation, we have inverted the slope in order to compare with our results. ${ }^{30}$ In the Appendix we show that although the slope of $P\left(L_{X} \mid M\right)$ is not exactly equal to the inverse of the slope of $P\left(M \mid L_{X}\right)$, the difference is small (also see Figure 2).

A representative (but not exhaustive) list of X-ray-based results is as follows.

1. Reiprich \& Böhringer (2002): $L_{\mathrm{X}} \propto M_{200}^{1.496 \pm 0.089}$ or $L_{\mathrm{X}} \propto$ $M_{200}^{1.652 \pm 0.085}$ depending on the fitting method (extended sample, Table 7 of their paper). The inverse slopes are $0.67 \pm 0.04$ and $0.60 \pm 0.03$, respectively.

2. Allen et al. (2003): $M_{200} \propto L_{\mathrm{X}}^{0.76_{-0.13}^{+0.16}}$

3. Popesso et al. (2005): $L_{\mathrm{X}} \propto M_{200}^{1.58 \pm 0.23}$. The inverse slope is $0.63 \pm 0.09$.

4. Chen et al. (2007) $: L_{\mathrm{X}} \propto M_{200}^{1.82 \pm 0.13}$. The inverse slope is $0.55 \pm 0.04$.

5. Pratt et al. (2009): $L_{1} \propto M_{Y}^{1.81 \pm 0.10}$ or $L_{1} \propto M_{Y}^{1.96 \pm 0.11}$ depending on the fitting method (Table 2 in their paper). The inverse slopes are $0.55 \pm 0.03$ and $0.51 \pm 0.029$.

6. Vikhlinin et al. (2009): $L_{\mathrm{X}} \propto M_{200}^{1.61 \pm 0.14}$. The inverse slope is $0.62 \pm 0.05$.

Figure 9 shows a comparison between X-ray-based estimates of the slope of the $M-L_{\mathrm{X}}$ relation and lensing-based results. As can be seen in this figure, most of the lensing and the $\mathrm{X}$-ray results are in excellent agreement with an average slope of $\alpha \sim 0.64$.

Evolution of scaling relations. Large surveys that will probe clusters up to $z=1$ will need a precise understanding of the redshift evolution in mass-observable relations. In this paper, we have shown that weak gravitational lensing is capable of meeting this challenge, and we have tested several evolution

${ }^{30}$ Errors on $y=\frac{1}{x}$ have been computed as $\Delta y=\left(\frac{1}{x}\right)^{2} \times \Delta x$. scenarios from $z \sim 0.2$ to $z \sim 0.9$. Our results are consistent with the evolution rate predicted by self-similarity, but our errors are still relatively large due to our small sample size at high redshift. Additional X-ray data would help improve the precision of this measurement. More precise measurements of the redshift evolution of X-ray scaling laws would also help constrain the physical processes that govern the heating and cooling of the ICM.

Self-calibration methods. Sufficiently large cluster surveys may be able to deal with the evolution and scatter in massobservable relations by internally calibrating for these uncertainties (the so-called "self-calibration method," Levine et al. 2002; Hu 2003; Majumdar \& Mohr 2004; Wang et al. 2004; Lima \& Hu 2005). This method treats all uncertainties as free parameters to be fitted along with the desired cosmological parameters. However, there are several drawbacks to this method. The first is that treating systematic errors in this manner weakens the final statistical constraints. The second is that self-calibration requires a parametric form for the scatter and evolution of scaling relations. Bias is introduced if this parameterization is incorrect.

This paper has showed that weak gravitational lensing can help constrain the actual form of the mass-observable relations as well as their evolution, even out to high redshifts $(z<1)$. Having a (direct observational) external constraint will help boost the accuracy achievable with self-calibration methods by reducing the number of parameters and by pinning down the correct parametric form. One important ingredient in the $M-L_{\mathrm{X}}$ relation, and for self-calibration exercises, is the scatter $\sigma_{\ln M}$. Stacked weak lensing measurements are not suitable for measuring the scatter and so individual lensing detections will be necessary for this task. Consequently, estimates of the scatter with lensing will be limited to high masses and moderate redshifts (see Figure 1). Space-based data will be optimal for scatter studies because high source densities will increase the mass and redshift range for which clusters can be directly probed. The challenge for lensing-based estimates of the scatter will be to ensure that the lensing errors are smaller than the intrinsic scatter, a condition that is probably not achieved at present. 
Nevertheless, one interesting point to note is that scatter will introduce a small amount of curvature in stacked lensing-based measurements of the $M-L_{\mathrm{X}}$ relation (see Figure 2). Therefore, it is possible that the scatter could be measured with future data by constraining the amount of curvature in $M-L_{\mathrm{X}}$ at high halo masses.

In conclusion, the field of weak gravitational has started to become a truly competitive tool for calibrating the relation between the total mass of groups and clusters of galaxies and their baryonic tracers, over a wide range of masses $\left(M_{200} \sim\right.$ $10^{13.5}-10^{15.5} h_{72}^{-1} M_{\odot}$ ) and up to $z=1$. At present, the slope of the $M-L_{\mathrm{X}}$ relation is constrained by lensing with a statistical significance that is comparable to X-ray studies (at the $\sim 5 \%$ level). Although further work will be necessary in order to compare both the slope and the normalization, it is encouraging to note that both lensing and X-rays studies are already in good agreement with respect to the slope of the $M-L_{\mathrm{X}}$ relation $(\alpha \sim 0.64)$. The observational foundation for the calibration of the mass-observable relations that are essential for cosmological studies with clusters of galaxies is clearly growing firmer.

We thank Uros Seljak, Reiko Nakajima, Henk Hoekstra, Rachel Mandelbaum, Chris Hirata, Masahiro Takada, and Satoshi Miyazaki for useful discussions. We thank Florian Pacaud for providing X-ray data for this analysis. We are also grateful to Eduardo Rozo and to Joel Bergé for providing comments on the manuscript and to an anonymous referee for insightful comments. A.L. acknowledges support from the Chamberlain Fellowship at LBNL and from the Berkeley Center for Cosmological Physics. J.P.K. acknowledges CNRS and CNES for support. J.P.K. and H.J.M.C.C. acknowledge support from the research grant ANR-07-BLAN-0228. The HST COSMOS Treasury program was supported through NASA grant HST-GO-09822. We thank Tony Roman, Denise Taylor, and David Soderblom for their assistance in planning and scheduling of the extensive COSMOS observations. We gratefully acknowledge the contributions of the entire COSMOS collaboration consisting of more than 70 scientists. More information on the COSMOS survey is available at http://cosmos.astro.caltech.edu/. It is a pleasure to acknowledge the excellent services provided by the NASA IPAC/IRSA staff (Anastasia Laity, Anastasia Alexov, Bruce Berriman, and John Good) in providing online archive and server capabilities for the COSMOS data sets.

\section{APPENDIX}

In this appendix, we derive the relation between $P\left(M \mid L_{X}, z\right)$ and $P\left(L_{X} \mid M, z\right)$ and show that a correction needs to be applied when comparing the slopes of the mean log luminosity and the mean log mass over a wide range in halo mass. The slope of the mean relation associated with $P\left(M \mid L_{X}, z\right)$ is noted $\alpha_{\text {lensing, }}$, and the slope of $P\left(L_{X} \mid M, z\right)$ is noted $\beta$. Partial aspects of this derivation can also be found in the appendix of Mandelbaum \& Seljak (2007).

Let $m \equiv \ln M$ and $l \equiv \ln L_{X}$, where $M$ represents the halo mass. Following Stanek et al. (2006), we assume that the conditional probability distribution of the luminosity given the mass is log-normal. In this case, $P(l \mid m, z)$ is Gaussian and we can write that

$$
P(l \mid m, z)=\frac{1}{\sigma_{l} \sqrt{2 \pi}} \times \exp \left(\frac{-\left[l-l_{0}(m, z)\right]^{2}}{2 \sigma_{l}^{2}}\right),
$$

where $l_{0}(m, z)$ is the mean log luminosity, and $\sigma_{l}$ is the scatter (also noted $\sigma_{\ln L_{X}}$ ). We assume that the scatter varies neither with mass, nor redshift ( $\sigma_{l}$ is constant) and that the mean log luminosity follows a power-law scaling relation with mass and with self-similar redshift evolution:

$$
\left\langle\ln L_{\mathrm{X}}\right\rangle \equiv l_{0}(m, z)=\beta m+(1+\beta) \ln E(z)+B .
$$

Let $n(M)$ represent the number of dark matter halos per unit volume with mass less than $M$. Locally, the differential mass function is a power law of the form $d n / d M \propto M^{-\gamma}=e^{-\gamma m}$. The probability of observing a halo of log-mass $m$ is $P(m)=$ $d n / d \ln M \propto e^{-(\gamma-1) m}$.

The weak lensing signal of halos stacked according to $L_{\mathrm{X}}$ depends on $P\left(M \mid L_{X}, z\right)$, the conditional probability distribution of the mass given the luminosity. Using Bayes theorem and ignoring those terms that only contribute to the overall normalization of $P(m \mid l, z)$, we can write that

$$
\begin{aligned}
P(m \mid l, z) & \propto P(l \mid m, z) P(m, z) \\
& \propto \exp \left(\frac{-\left[l-l_{0}(m, z)\right]^{2}}{2 \sigma_{l}^{2}}-(\gamma-1) m\right) .
\end{aligned}
$$

By using Equation (A2) to develop the expression within the exponential and by completing the square, we obtain that

$$
P(m \mid l, z) \propto \exp \left(\frac{-\left[m-m_{0}(l, z)\right]^{2}}{2\left(\sigma_{l} \beta^{-1}\right)^{2}}\right),
$$

where $\langle\ln M\rangle \equiv m_{0}(l, z)=(1 / \beta) l-(1+1 / \beta) \ln E(z)-$ $B / \beta-\sigma_{m}^{2}(\gamma-1)$. In other terms, $P(m \mid l, z)$ is Gaussian, and $P\left(M \mid L_{X}, z\right)$ is log-normal with a dispersion in mass equal to $\sigma_{m}=\sigma_{l} / \beta$ and with a mean log mass that follows a power law of with a slope of $\alpha_{\text {lensing }}=1 / \beta$. The mean log-mass, $m_{0}(l, z)$, can be obtained by solving for the mass in Equation (A2) with the addition of an extra term. In this sense, there is a bias between $m_{0}(l, z)$ and the true mean mass which is equal to $b_{m}=\sigma_{m}^{2}(\gamma-1)$, and this bias scales linearly with $\gamma-1$, where $\gamma$ is the slope of the mass function. Because, $(\gamma-1)$ is always positive, this bias causes $m_{0}(l, z)$ to be biased low relative to the true mean mass.

Both mass and luminosity are often expressed in units of logarithm to the base 10 rather than in units of natural logarithm. In this case, the scatter in mass at fixed luminosity is equal to $\sigma_{\log _{10} M} \equiv \sigma_{m 10}=\sigma_{m} / \ln (10)$, and the bias is equal to $b_{m 10}=\sigma_{m 10}^{2}(\gamma-1) \ln (10)=\sigma_{m}^{2}(\gamma-1) / \ln (10)$. Note that there is an extra factor of $\ln (10)$ in the expression of the bias due to the fact that $P\left(\log _{10} M\right)=d n / d \log _{10} M \propto M^{-(\gamma-1)}=$ $e^{-(\gamma-1) \ln (10) \log _{10}(M)}$

We have demonstrated that $\alpha_{\text {lensing }}=1 / \beta$ when the halo mass function is locally a power law. In reality, the slope of the mass function varies with both mass and redshift. At $z=0.2$, the slope of the mass function varies from $\gamma \sim 2$ at $M_{200} \sim 10^{13} h_{72}^{-1} M_{\odot}$ to $\gamma \sim 5$ at $M_{200} \sim 10^{15} h_{72}^{-1} M_{\odot}$. The fact that the bias depends on $\gamma$ will result in a change in slope and $\alpha_{\text {lensing }}=1 / \beta$ will no longer be valid. Instead, there will be a correction factor between the slope derive by stacked weak lensing, $\alpha_{\text {lensing }}$, and $1 / \beta$, and we will now show how this correction factor can be roughly estimated.

Let $m_{1}$ and $m_{2}$ represent two distinct halo masses in units of logarithm to the base 10 . The local slope of the mass function at each of these masses is noted as $\gamma_{1}$ and $\gamma_{2}$ and the biases are noted as $b_{m 1}=\sigma_{m}^{2} \gamma_{1} / \ln (10)$ and $b_{m 2}=\sigma_{m 10}^{2} \gamma_{2} / \ln (10)$. 
The slope that is derived via stacked weak lensing over the mass range $\left[m_{1}, m_{2}\right]$ is equal to $\alpha_{\text {lensing }}=F / \beta$, where $F=$ $1-\left(b_{m 1}-b_{m 2}\right) /\left(m_{1}-m_{2}\right)$. For $z=0.2, \sigma_{m}=0.25$ $\left(\sigma_{m 10} \sim 0.109\right), M_{1} \sim 10^{13} h_{72}^{-1} M_{\odot}$ and $M_{2} \sim 10^{15} h_{72}^{-1} M_{\odot}$, we have $b_{m 1} \sim 0.03$ and $b_{m 2} \sim 0.11$ (when $m$ is expressed in $\log _{10}$ units). The difference between $1 / \beta$ and $\alpha_{\text {lensing }}$ over this mass range is therefore roughly $\alpha_{\text {lensing }} \sim 0.96 / \beta$.

\section{REFERENCES}

Albrecht, A., et al. 2006, arXiv:astro-ph/0609591

Allen, S. W., Schmidt, R. W., Fabian, A. C., \& Ebeling, H. 2003, MNRAS, 342, 287

Arnaud, M., Pointecouteau, E., \& Pratt, G. W. 2005, A\&A, 441, 893

Bahcall, N. A., Hao, L., Bode, P., \& Dong, F. 2004, ApJ, 603, 1

Bardeau, S., Soucail, G., Kneib, J.-P., Czoske, O., Ebeling, H., Hudelot, P., Smail, I., \& Smith, G. P. 2007, A\&A, 470, 449

Bartelmann, M. 1996, A\&A, 313, 697

Benson, B. A., Church, S. E., Ade, P. A. R., Bock, J. J., Ganga, K. M., Henson,

C. N., \& Thompson, K. L. 2004, ApJ, 617, 829

Bergé, J., et al. 2008, MNRAS, 385, 695

Bertin, E., \& Arnouts, S. 1996, A\&AS, 117, 393

Bildfell, C., Hoekstra, H., Babul, A., \& Madhavi, A. 2008, MNRAS, 389, 1637

Böhringer, H., et al. 2000, ApJS, 129, 435

Böhringer, H., et al. 2004, A\&A, 425, 367

Bruzual, G., \& Charlot, S. 2003, MNRAS, 344, 1000

Bullock, J. S., Kolatt, T. S., Sigad, Y., Somerville, R. S., Kravtsov, A. V., Klypin, A. A., Primack, J. R., \& Dekel, A. 2001, MNRAS, 321, 559

Bundy, K. 2006a, PhD thesis, California Institute of Technology

Bundy, K., et al. 2006b, ApJ, 651, 120

Capak, P., et al. 2007, ApJS, 172, 99

Cappelluti, N., et al. 2009, A\&A, 497, 635

Carlstrom, J. E., Holder, G. P., \& Reese, E. D. 2002, ARA\&A, 40, 643

Chen, Y., Reiprich, T. H., Böhringer, H., Ikebe, Y., \& Zhang, Y.-Y. 2007, A\&A, 466, 805

Cohn, J. D., \& White, M. 2009, MNRAS, 393, 393

de Putter, R., \& White, M. 2005, New Astron., 10, 676

Eisenhardt, P. R. M., et al. 2008, ApJ, 684, 905

Elvis, M., et al. 2009, ApJS, 184, 158

Ettori, S., et al. 2004, MNRAS, 354, 111

Finoguenov, A., et al. 2007, ApJS, 172, 182

Finoguenov, A., et al. 2009, MNRAS, submitted (arXiv:0912.0039)

Gerke, B. F., et al. 2005, ApJ, 625, 6

Gladders, M. D., \& Yee, H. K. C. 2005, ApJS, 157, 1

Gnedin, O. Y., Kravtsov, A. V., Klypin, A. A., \& Nagai, D. 2004, ApJ, 616, 16

Haiman, Z., Mohr, J. J., \& Holder, G. P. 2001, ApJ, 553, 545

Hamana, T., Takada, M., \& Yoshida, N. 2004, MNRAS, 350, 893

Hasinger, G., et al. 2001, A\&A, 365, L45

Hasinger, G., et al. 2007, ApJS, 172, 29

Heymans, C., et al. 2006, MNRAS, 368, 1323

Hinshaw, G., et al. 2009, ApJS, 180, 225

Hoekstra, H. 2003, MNRAS, 339, 1155

Hoekstra, H. 2007, MNRAS, 379, 317

Hu, W. 2003, Phys. Rev. D, 67, 081304

Ilbert, O., et al. 2006, A\&A, 457, 841

Ilbert, O., et al. 2009, ApJ, 690, 1236

Jeltema, T. E., Canizares, C. R., Bautz, M. W., \& Buote, D. A. 2005, ApJ, 624, 606

Johnston, D. E., et al. 2007, arXiv:0709.1159

Kaiser, N. 1986, MNRAS, 222, 323

Knobel, C., et al. 2009, ApJ, 697, 1842

Koekemoer, A. M., et al. 2007, ApJS, 172, 196

Koester, B. P., et al. 2007, ApJ, 660, 239

Kotov, O., \& Vikhlinin, A. 2005, ApJ, 633, 781

Kravtsov, A. V., Vikhlinin, A., \& Nagai, D. 2006, ApJ, 650, 128

Leauthaud, A., et al. 2007, ApJS, 172, 219

Levine, E. S., Schulz, A. E., \& White, M. 2002, ApJ, 577, 569

Lilly, S. J., et al. 2007, ApJS, 172, 70

Lima, M., \& Hu, W. 2005, Phys. Rev. D, 72, 043006

Lumb, D. H., et al. 2004, A\&A, 420, 853

Macciò, A. V., Dutton, A. A., van den Bosch, F. C., Moore, B., Potter, D., \& Stadel, J. 2007, MNRAS, 378, 55
Madhavi, A., Hoekstra, H., Babul, A., \& Henry, J. P. 2008, MNRAS, 384, 1567

Majumdar, S., \& Mohr, J. J. 2004, ApJ, 613, 41

Mandelbaum, R., \& Seljak, U. 2007, JCAP, 6, 24

Mandelbaum, R., Seljak, U., Cool, R. J., Blanton, M., Hirata, C. M., \& Brinkmann, J. 2006a, MNRAS, 372, 758

Mandelbaum, R., Seljak, U., Kauffmann, G., Hirata, C. M., \& Brinkmann, J. 2006b, MNRAS, 368, 715

Mandelbaum, R., Tasitsiomi, A., Seljak, U., Kravtsov, A. V., \& Wechsler, R. H. 2005, MNRAS, 362, 1451

Mandelbaum, R., et al. 2008, MNRAS, 386, 781

Marian, L., \& Bernstein, G. M. 2006, Phys. Rev. D, 73, 123525

Markevitch, M. 1998, ApJ, 504, 27

Massey, R., Stoughton, C., Leauthaud, A., Rhodes, J., Koekemoer, A., Ellis, R., \& Shaghoulian, E. 2009, arXiv:0909.507

Massey, R., et al. 2007a, MNRAS, 376, 13

Massey, R., et al. 2007b, Nature, 445, 286

Maughan, B. J. 2007, ApJ, 668, 772

McCracken, H. J., et al. 2009, arXiv:0910.2705

Metzler, C. A., White, M., \& Loken, C. 2001, ApJ, 547, 560

Miller, C. J., et al. 2005, AJ, 130, 968

Miralda-Escude, J. 1991, ApJ, 370, 1

Miyazaki, S., Hamana, T., Ellis, R. S., Kashikawa, N., Massey, R. J., Taylor, J., \& Refregier, A. 2007, ApJ, 669, 714

Motl, P. M., Hallman, E. J., Burns, J. O., \& Norman, M. L. 2005, ApJ, 623, L63

Nagai, D. 2006, ApJ, 650, 538

Nagai, D., Kravtsov, A. V., \& Vikhlinin, A. 2007, ApJ, 668, 1

Navarro, J. F., Frenk, C. S., \& White, S. D. M. 1997, ApJ, 490, 493

Popesso, P., Biviano, A., Böhringer, H., Romaniello, M., \& Voges, W. 2005, A\&A, 433, 431

Pratt, G. W., Croston, J. H., Arnaud, M., \& Boehringer, H. 2009, A\&A, 498, 361

Rapetti, D., Allen, S. W., Mantz, A., \& Ebeling, H. 2009, arXiv:0911.1787

Reiprich, T. H., \& Böhringer, H. 2002, ApJ, 567, 716

Rhodes, J., Refregier, A., \& Groth, E. J. 2000, ApJ, 536, 79

Rhodes, J. D., et al. 2007, ApJS, 172, 203

Rozo, E., Dodelson, S., \& Frieman, J. A. 2004, Phys. Rev. D, 70, 083008

Rozo, E., et al. 2009, ApJ, 699, 768

Rykoff, E. S., et al. 2008, MNRAS, 387, L28

Schmidt, F., Lima, M., Oyaizu, H., \& Hu, W. 2009, Phys. Rev. D, 79, 083518

Scoville, N., et al. 2007, ApJS, 172, 1

Sellwood, J. A., \& McGaugh, S. S. 2005, ApJ, 634, 70

Shaw, L. D., Holder, G. P., \& Bode, P. 2008, ApJ, 686, 206

Sheldon, E. S., et al. 2009, ApJ, 703, 2217

Stanek, R., Evrard, A. E., Böhringer, H., Schuecker, P., \& Nord, B. 2006, ApJ, 648,956

Stanford, S. A., et al. 2006, ApJ, 646, L13

Staniszewski, Z., et al. 2009, ApJ, 701, 32

Sunyaev, R. A., \& Zeldovich, Y. B. 1970, Ap\&SS, 7, 3

Sunyaev, R. A., \& Zeldovich, Y. B. 1972, Comments Astrophys. Space Phys., 4, 173

Tinker, J., Kravtsov, A. V., Klypin, A., Abazajian, K., Warren, M., Yepes, G., Gottlöber, S., \& Holz, D. E. 2008, ApJ, 688, 709

Vale, C., \& White, M. 2006, New Astron., 11, 207

Vikhlinin, A., McNamara, B. R., Forman, W., Jones, C., Quintana, H., \& Hornstrup, A. 1998, ApJ, 502, 558

Vikhlinin, A., et al. 2007, in Heating Versus Cooling in Galaxies and Clusters of Galaxies, ed. H. Böhringer, G. W. Pratt, A. Finoguenov, \& P. Schuecker (Berlin: Springer), 48

Vikhlinin, A., et al. 2009, ApJ, 692, 1033

Voit, G. M. 2005, Rev. Mod. Phys., 77, 207

Wang, L., \& Steinhardt, P. J. 1998, ApJ, 508, 483

Wang, S., Khoury, J., Haiman, Z., \& May, M. 2004, Phys. Rev. D, 70, 123008

Wechsler, R. H., Bullock, J. S., Primack, J. R., Kravtsov, A. V., \& Dekel, A. 2002, ApJ, 568, 52

White, M., Hernquist, L., \& Springel, V. 2002, ApJ, 579, 16

White, S. D. M., Efstathiou, G., \& Frenk, C. S. 1993, MNRAS, 262, 1023

Wilson, G., Kaiser, N., Luppino, G. A., \& Cowie, L. L. 2001, ApJ, 555, 572

Wright, C. O., \& Brainerd, T. G. 2000, ApJ, 534, 34

Yoo, J., Tinker, J. L., Weinberg, D. H., Zheng, Z., Katz, N., \& Davé, R. 2006, ApJ, 652, 26

Zhang, Y.-Y., Finoguenov, A., Böhringer, H., Kneib, J.-P., Smith, G. P., Kneissl, R., Okabe, N., \& Dahle, H. 2008, A\&A, 482, 451

Zhao, D. H., Jing, Y. P., Mo, H. J., \& Boerner, G. 2009, ApJ, 707, 354 